\title{
Existence of strong solutions for the motion of an elastic structure in an incompressible viscous fluid
}

\author{
Muriel BOULAKIA \\ Université Pierre et Marie Curie Paris 6, UMR 7598, Laboratoire Jacques-Louis Lions, 75005 \\ Paris, France \\ E-mail:boulakia@ann.jussieu.fr \\ ERICA L. SCHWINDT \\ Dpto. de Ingeniería Matemática, Facultad de Ciencias Físicas y Matemáticas, Universidad de \\ Chile, Santiago, Chile; Inria, Villers-lès-Nancy, 54600, France; Université de Lorraine, IECN, \\ U.M.R. 7502, Vandoeuvre-lès-Nancy, 54506, France; E-mail: leris98@gmail.com
}

TAKÉO TAKAHASHI

Inria, Villers-lès-Nancy, 54600, France; Université de Lorraine, IECN, U.M.R. 7502,

Vandoeuvre-lès-Nancy, 54506, France; CNRS, IECN, U.M.R. 7502, Vandoeuvre-lès-Nancy, 54506, France

E-mail:Takeo.Takahashi@inria.fr

[Received 8 February 2011 and in revised form 15 March 2012]

\begin{abstract}
In this paper we study a three-dimensional fluid-structure interaction problem. The motion of the fluid is modeled by the Navier-Stokes equations and we consider for the elastic structure a finitedimensional approximation of the equation of linear elasticity. The time variation of the fluid domain is not known a priori, so we deal with a free boundary value problem. Our main result yields the local in time existence and uniqueness of strong solutions for this system.
\end{abstract}

2010 Mathematics Subject Classification: Primary 74F10, 76D03; Secondary 35R35, 35Q30, 37N15, $76 \mathrm{D} 05$.

Keywords: Fluid-structure interaction, existence and uniqueness of strong solutions, incompressible Navier-Stokes equations, deformable structure

\section{Introduction}

We consider the interaction between a viscous incompressible fluid and an elastic structure immersed in the fluid. We aim to study the coupled system of equations modeling the motions of the fluid and of the structure. A classical model for the fluid is the Navier-Stokes equations (see system (7)); for the elastic structure, assuming that the deformation remains small, we can consider the model of linear elasticity. When we couple these two systems of partial differential equations, we obtain a complex system and the study of its well-posedness contains several difficulties. One of them is coming from the fact that we gather two systems of different natures. This could be observed by linearizing the system: we obtain a system coupling a parabolic system with an hyperbolic system. Another general difficulty to study this fluid-structure interaction problem comes from the fact that the fluid domain is moving and unknown (it is a free boundary problem). In particular, to apply "classical" procedures on cylindrical domain, it is convenient to use a change of variables 
for the fluid equations (the structure equations are already written in Lagrangian coordinates). But such a change of variables is constructed from the solution and thus the solution needs to be regular enough to get a convenient change of variables.

The well-posedness for the coupling of these two systems is studied in [8] (for the linear case) and in [5] (for the general case). The combination of the two difficulties presented above leads the authors of [5] to consider the existence of regular solutions for the linearized problem (the velocity of the fluid is $\boldsymbol{L}^{2}$ in time with value in $\boldsymbol{H}^{3}$ in space). In that case, they need more regularity for the initial data: the initial condition is for instance $\boldsymbol{H}^{5}$ in space. This loss of regularity is not very satisfactory but is inherent to this coupling between two systems of different natures.

In order to avoid this loss of regularity, one can choose to consider an approximation of the previous system. Some approximations were already tackled in the literature in particular to obtain existence of weak solutions. In that case, it is very important to obtain some regularity on the elastic deformations which define the fluid domain, at least to give a sense to the equations of the fluid. Two strategies have been considered: one could add a regularizing term in the equations of linear elasticity (see [1]) or one could approximate the equations of linear elasticity by a finite dimensional system (see [7]). In each case, the authors of [1] and of [7] have obtained the existence of weak solutions (up to a contact).

In this article, our main result is the existence and uniqueness of strong solution for a system coupling the Navier-Stokes system with a finite dimensional approximation of the linear elasticity (i.e., a system similar to the one considered in [7]).

Let us present here the system we consider. We denote by $\boldsymbol{u}$ and $p$ the velocity and the pressure of the fluid, and by $\xi$ the elastic deformation of the structure. We also denote by $\Omega$ the domain containing the fluid and the structure, by $\Omega_{F}(t)$ the fluid domain and by $\Omega_{S}(t)$ the structure domain at time $t$. These three domains are assumed to be bounded, open and connected subsets of $\mathbb{R}^{3}$. We also suppose that $\partial \Omega$ is of class $C^{2}$ and that at initial time, $\partial \Omega_{S}(0)$ is of class $C^{3}$. Finally, we assume that the elastic body is immersed into the fluid: $\overline{\Omega_{S}(t)} \subset \Omega$ and $\Omega_{F}(t)=\Omega \backslash \overline{\Omega_{S}(t)}$.

Let us first introduce the usual equation of linear elasticity which is valid for small deformations:

$$
\frac{\partial^{2} \xi}{\partial t^{2}}-\operatorname{div}(\pi(\xi))=\mathbf{0} \text { in } \Omega_{S}(0) .
$$

The stress tensor is given by (see [3])

$$
\pi(\xi)=\lambda \operatorname{tr}(\varepsilon(\xi)) \mathrm{I}+2 \mu \varepsilon(\xi)
$$

where $\boldsymbol{\varepsilon}(\boldsymbol{\xi})=\frac{1}{2}\left(\nabla \boldsymbol{\xi}+(\nabla \xi)^{t}\right)$ and Lamé constants $\lambda$ and $\mu$ are such that $\mu>0$ and $\lambda \geqslant 0$. Through the fixed interface $\partial \Omega_{S}(0)$ between the fluid and the structure, it is assumed that the normal component of the stress tensors is continuous:

$$
(\boldsymbol{\pi}(\boldsymbol{\xi}))(t, \boldsymbol{y}) \boldsymbol{n}_{\boldsymbol{y}}=\boldsymbol{\sigma}(\boldsymbol{u}, p)(t, \boldsymbol{\varphi}(t, \boldsymbol{y})) \operatorname{Cof}(\nabla \boldsymbol{\varphi}) \boldsymbol{n}_{\boldsymbol{x}} \quad \text { on } \quad \partial \Omega_{S}(0) .
$$

Here $\boldsymbol{n}_{\boldsymbol{y}}$ is the outward-pointing unit normal vector along $\partial \Omega_{S}(0), \boldsymbol{n}_{\boldsymbol{x}}$ is the outward-pointing unit normal vector along $\partial \Omega_{S}(t)$ and

$$
\boldsymbol{\sigma}(\boldsymbol{u}, p)=-p \mathbf{I d}+2 v \boldsymbol{\varepsilon}(\boldsymbol{u})
$$

is the Cauchy stress tensor associated to the fluid. We have also denoted by $\varphi$ the elastic displacement defined by

$$
\varphi(t, y):=y+\xi(t, y)
$$




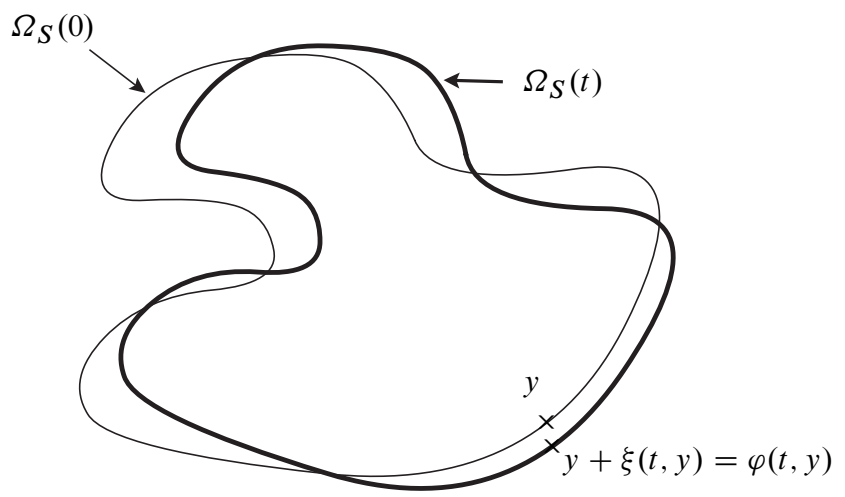

FIG. 1. Elastic deformation

(see Figure 1). To simplify, we assume that at initial time, there is no deformation, thus we have $\varphi(0, y)=y$.

In this paper, we do not consider the complete equation of linear elasticity written above, but only a finite approximation of Galerkin type: we assume that $\xi$ can be written as a linear combination of a family of $N_{0}$ functions. Let us note that we do not choose here a particular family as it is done in [7] where the authors use a family of eigenmodes associated to the elasticity equation.

We can notice that the incompressibility of the fluid enforces the deformation $\xi$ to satisfy some compatibility conditions. Indeed, at the interface between the fluid and the structure, the normal components of the velocity of the fluid and of the structure are equal. Combined with the incompressibility of the fluid, it yields

$$
\operatorname{div}(\boldsymbol{u})=0 \quad \text { in } \quad \Omega_{S}(t), \quad \frac{\partial \xi}{\partial t}\left(t, \varphi^{-1}(t, \boldsymbol{x})\right) \cdot \boldsymbol{n}_{\boldsymbol{x}}=\boldsymbol{u}(t, \boldsymbol{x}) \cdot \boldsymbol{n}_{\boldsymbol{x}} \quad \text { on } \quad \partial \Omega_{S}(t) .
$$

Thus, if we assume that $\boldsymbol{u}=\mathbf{0}$ on $\partial \Omega$, it implies the following non linear condition on $\boldsymbol{\xi}$ :

$$
\int_{\partial \Omega_{S}(t)} \frac{\partial \xi}{\partial t}\left(t, \varphi^{-1}(t, \boldsymbol{x})\right) \cdot \boldsymbol{n}_{\boldsymbol{x}} d \boldsymbol{\gamma}_{\boldsymbol{x}}=0
$$

By this way, the Galerkin approximation of $\boldsymbol{\xi}$ has to satisfy this condition. Let $\boldsymbol{\xi}_{1}, \ldots, \boldsymbol{\xi}_{N_{0}}\left(N_{0} \geqslant 1\right)$ be a given orthonormal family in $\boldsymbol{L}^{2}\left(\Omega_{S}(0)\right)$ which satisfies

$$
\xi_{i} \in \boldsymbol{H}^{3}\left(\Omega_{S}(0)\right), \quad \int_{\partial \Omega_{S}(0)} \boldsymbol{\xi}_{i} \cdot \boldsymbol{n}_{\boldsymbol{y}} d \boldsymbol{\gamma}_{\boldsymbol{y}}=0 \quad i=1,2, \ldots, N_{0}
$$

If $\boldsymbol{\xi}$ is a linear combination of $\boldsymbol{\xi}_{1}, \ldots, \boldsymbol{\xi}_{N_{0}}$, it satisfies (3) at initial time, but there is no reason that it should be true for $t>0$. A remedy to this problem is to add an extra function $\xi_{0}$ in order to satisfy (3). More precisely, let $\boldsymbol{\xi}_{0} \in \boldsymbol{H}^{3}\left(\Omega_{S}(0)\right)$ be a lifting of the unit outward normal on $\partial \Omega_{S}(0)$. Then, it can been proved (see Lemma 2.1 below) that there exists a ball $B\left(\mathbf{0}, r_{1}\right)$ of $\mathbb{R}^{N_{0}}$ and a function $\phi: B\left(\mathbf{0}, r_{1}\right) \rightarrow \mathbb{R}$ such that for any function (regular enough) $\left(\alpha_{1}(t), \ldots, \alpha_{N_{0}}(t)\right) \in B\left(\mathbf{0}, r_{1}\right)$, the associated deformation

$$
\boldsymbol{\xi}(t, \boldsymbol{y}):=\sum_{i=0}^{N_{0}} \alpha_{i}(t) \xi_{i}(\boldsymbol{y}), \quad \text { with } \quad \alpha_{0}(t)=\phi\left(\alpha_{1}(t), \ldots, \alpha_{N_{0}}(t)\right),
$$


satisfies (3). Using this construction, we can also define a family $\left(\widehat{\boldsymbol{\xi}}_{1}(t), \ldots, \widehat{\boldsymbol{\xi}}_{N_{0}}(t)\right)$ where for all $i, \widehat{\xi_{i}}$ is a linear combination of $\boldsymbol{\xi}_{i}$ and $\boldsymbol{\xi}_{0}$ (see (19)) so that if we write

$$
\frac{\partial \boldsymbol{\xi}}{\partial t}(t, \boldsymbol{y})=\sum_{i=1}^{N_{0}} \beta_{i}(t) \widehat{\boldsymbol{\xi}}_{i}(t, \boldsymbol{y}), \quad \text { with } \quad \beta_{i}=\alpha_{i}^{\prime},
$$

then (3) is always satisfied.

We are now in position to introduce the system we study in this article: the system of equations for the fluid is the classical Navier-Stokes system

$$
\begin{cases}\frac{\partial \boldsymbol{u}}{\partial t}-v \boldsymbol{\Delta} \boldsymbol{u}+\nabla p+(\boldsymbol{u} \cdot \nabla) \boldsymbol{u}=\mathbf{0} & \text { in } \Omega_{F}(t) \\ \operatorname{div}(\boldsymbol{u})=0 & \text { in } \Omega_{F}(t) \\ \boldsymbol{u}=\mathbf{0} & \text { on } \partial \Omega \\ \frac{\partial \boldsymbol{\xi}}{\partial t}(t, \boldsymbol{y})=\boldsymbol{u}(t, \varphi(t, \boldsymbol{y})) & \text { on } \partial \Omega_{S}(0)\end{cases}
$$

and the equations for the structure are a Galerkin approximation of (1)-(2)

$$
\begin{aligned}
\int_{\Omega_{S}(0)} \frac{\partial^{2} \boldsymbol{\xi}}{\partial t^{2}} \cdot \widehat{\boldsymbol{\xi}}_{i} d \boldsymbol{y}+ & 2 \mu \int_{\Omega_{S}(0)} \boldsymbol{\varepsilon}(\boldsymbol{\xi}): \boldsymbol{\varepsilon}\left(\widehat{\boldsymbol{\xi}}_{i}\right) d \boldsymbol{y}+\lambda \int_{\Omega_{S}(0)} \operatorname{div}(\boldsymbol{\xi}) \operatorname{div}\left(\widehat{\boldsymbol{\xi}}_{i}\right) d \boldsymbol{y} \\
& =\int_{\partial \Omega_{S}(t)} \boldsymbol{\sigma}(\boldsymbol{u}, p)(t, \boldsymbol{x}) \boldsymbol{n}_{\boldsymbol{x}} \cdot \widehat{\boldsymbol{\xi}}_{i}\left(t, \boldsymbol{\varphi}^{-1}(t, \boldsymbol{x})\right) d \boldsymbol{\gamma}_{\boldsymbol{x}}, \quad i=1, \ldots, N_{0}
\end{aligned}
$$

with $\boldsymbol{\xi}$ satisfying (5) and thus with $\frac{\partial \boldsymbol{\xi}}{\partial t}$ satisfying (6). We recall that $\varphi(t, \boldsymbol{y}):=\boldsymbol{y}+\boldsymbol{\xi}(t, \boldsymbol{y})$ and we assume that $\varphi(t)$ is invertible (which is the case for $r_{1}$ small enough). This system is completed by the initial conditions

$$
\boldsymbol{u}_{\left.\right|_{t=0}}=\boldsymbol{u}^{0} \quad \text { in } \quad \Omega_{F}(0), \quad \boldsymbol{\xi}_{\left.\right|_{t=0}}=\mathbf{0} \quad \text { in } \quad \Omega_{S}(0),\left.\quad \frac{\partial \boldsymbol{\xi}}{\partial t}\right|_{t=0}=\xi^{1} \quad \text { in } \quad \Omega_{S}(0),
$$

with

$$
\xi^{1}=\sum_{i=1}^{N_{0}} \beta_{i}^{0} \xi_{i}
$$

where $\beta_{i}^{0}=\beta_{i}(0)$. Note that

$$
\widehat{\xi}_{i}(0, y)=\xi_{i}(y) \text { for all } i \in\left\{1, \ldots, N_{0}\right\} .
$$

For simplicity of notation, in what follows, we set

$$
\begin{aligned}
\Omega_{F} & :=\Omega_{F}(0), \\
\Omega_{S} & :=\Omega_{S}(0) .
\end{aligned}
$$

Now, we define the function spaces $\boldsymbol{L}^{2}\left(0, T ; \boldsymbol{H}^{2}\left(\Omega_{F}(t)\right)\right), \boldsymbol{C}\left([0, T] ; \boldsymbol{H}^{1}\left(\Omega_{F}(t)\right)\right)$ and $\boldsymbol{H}^{1}\left(0, T ; \boldsymbol{L}^{2}\left(\Omega_{F}(t)\right)\right)$, which will be extensively used in the sequel. Suppose there exists a function 
$\boldsymbol{\psi} \in \boldsymbol{H}^{2}\left(0, T ; \boldsymbol{H}^{3}\left(\Omega_{F}\right)\right)$ such that $\boldsymbol{\psi}(t, \cdot): \Omega_{F} \rightarrow \Omega_{F}(t)$ is a diffeomorphism. For all functions $\boldsymbol{w}(t, \cdot): \Omega_{F}(t) \rightarrow \mathbb{R}^{3}$, we denote $\boldsymbol{W}(t, \boldsymbol{y})=\boldsymbol{w}(t, \boldsymbol{\psi}(t, \boldsymbol{y}))$. Then the function spaces introduced above are defined by

$$
\begin{aligned}
\boldsymbol{L}^{2}\left(0, T ; \boldsymbol{H}^{2}\left(\Omega_{F}(t)\right)\right) & =\left\{\boldsymbol{w}: \boldsymbol{W} \in \boldsymbol{L}^{2}\left(0, T ; \boldsymbol{H}^{2}\left(\Omega_{F}\right)\right)\right\}, \\
\boldsymbol{C}\left([0, T] ; \boldsymbol{H}^{1}\left(\Omega_{F}(t)\right)\right) & =\left\{\boldsymbol{w}: \boldsymbol{W} \in \boldsymbol{C}\left([0, T] ; \boldsymbol{H}^{1}\left(\Omega_{F}\right)\right)\right\}, \\
\boldsymbol{H}^{1}\left(0, T ; \boldsymbol{L}^{2}\left(\Omega_{F}(t)\right)\right) & =\left\{\boldsymbol{w}: \boldsymbol{W} \in \boldsymbol{H}^{1}\left(0, T ; \boldsymbol{L}^{2}\left(\Omega_{F}\right)\right)\right\} .
\end{aligned}
$$

Set

$$
Ð(t):=\boldsymbol{L}^{2}\left(0, T ; \boldsymbol{H}^{2}\left(\Omega_{F}(t)\right)\right) \cap \boldsymbol{C}\left([0, T] ; \boldsymbol{H}^{1}\left(\Omega_{F}(t)\right)\right) \cap \boldsymbol{H}^{1}\left(0, T ; \boldsymbol{L}^{2}\left(\Omega_{F}(t)\right)\right),
$$

and

$$
Ð=Ф(0)
$$

Our main result is the following:

THEOREM 1.1 Assume that $\boldsymbol{u}^{0} \in \boldsymbol{H}^{1}\left(\Omega_{F}\right)$ and $\left\{\beta_{i}^{0}\right\}_{i=1}^{N_{0}} \subset \mathbb{R}$ satisfy the following conditions:

$$
\left\{\begin{array}{lll}
\operatorname{div}\left(\boldsymbol{u}^{0}\right)=0 & \text { in } & \Omega_{F}, \\
\boldsymbol{u}^{0}=\mathbf{0} & \text { on } & \partial \Omega \\
\boldsymbol{u}^{0}=\boldsymbol{\xi}^{1} & \text { on } & \partial \Omega_{S} \\
\operatorname{dist}\left(\Omega_{S}, \partial \Omega\right)>0 . & &
\end{array}\right.
$$

Then, there exists a time $T>0$ such that the system (7), (8), (9) and (10) admits a unique solution (up to a constant for $p$ )

$$
\begin{gathered}
(\boldsymbol{u}, p) \in \boxplus(t) \times L^{2}\left(0, T ; H^{1}\left(\Omega_{F}(t)\right)\right), \\
\alpha_{1}, \ldots, \alpha_{N_{0}} \in H^{2}(0, T) .
\end{gathered}
$$

Let us give some remarks on the main result. First, one can see that the existence of the pressure is obtained up to a constant as in the case of a fluid alone or a fluid coupled with a rigid body. In the case of a deformable structure, the pressure of the fluid may be defined uniquely. This comes from the incompressibility of the fluid and the boundary condition (see, for instance, [11] for more details). Here, the choice of our decomposition of the elastic deformation and elastic velocity (see (5) and (6)) leads to the fact that the pressure is still defined up to a constant. Second, as already mentioned above, there are few results concerning the well-posedness of a system modeling the motion of an elastic structure in a viscous incompressible fluid. The existence of weak solutions for our system was obtained by [7] whereas the existence of weak solutions when adding a regularizing term in the equation of elasticity was proved in [1]. Concerning strong solutions, we only know the result of [5] (and [8] for the linear case). In [5], a local in time result is obtained for strong solution. They also obtained uniqueness by considering more regular solutions. In this latter paper, the hypotheses on the initial data to get existence are quite strong: the initial velocity of the fluid must be in $\boldsymbol{H}^{5}\left(\Omega_{F}(0)\right)$ and the initial velocity of the elastic structure must be in $\boldsymbol{H}^{2}\left(\Omega_{S}(0)\right)$. 
This implies in particular many compatibility conditions at $t=0$. Moreover, to obtain also the uniqueness, the authors have to add more hypotheses on the regularity of the initial conditions: the initial velocity of the fluid must be in $\boldsymbol{H}^{7}\left(\Omega_{F}(0)\right)$ and the initial velocity of the elastic structure must be in $\boldsymbol{H}^{4}\left(\Omega_{S}(0)\right)$. In the case where we consider a rigid body instead of an elastic structure, the corresponding system was studied in many papers: see, for instance, [4, 6, 8, 10, 12, 13, 15, 16]. The existence of weak and strong solutions was proved with similar regularity hypotheses as in the case of the Navier-Stokes system. Here as in the case of a rigid body, the velocity of the deformation is assumed to be finite-dimensional, but at the contrary to the case of a rigid body, the generators of the velocity of deformation depends on time (the family $\left(\widehat{\boldsymbol{\xi}}_{i}(t)\right)_{1 \leqslant i \leqslant N_{0}}$ ). Moreover, the change of variables used to transform the problem on a cylindrical domain is more technical: in the case of a rigid body, the idea was only to extend (and truncate) the translation and the rotation of the rigid body.

Our strategy is to construct a change of variables to transform $\Omega_{F}(t)$ onto $\Omega_{F}$ (Section 2) and to use this change of variables to write the system (7), (8), (9) and (10) on a cylindrical domain (Section 3). We obtain a nonlinear coupled system with variable coefficients. The idea is to write it in the form

$$
\mathbf{Z}^{\prime}=A \mathbf{Z}+\mathbf{R}(\mathbf{Z})
$$

where $\mathbf{Z}$ is $(\boldsymbol{u}, \boldsymbol{\beta})$, with $\boldsymbol{\beta}=\left(\beta_{1}, \ldots, \beta_{N_{0}}\right)$. In $\mathbf{R}(\mathbf{Z})$, we put all the coefficients coming from the change of variables and the nonlinear terms coming from the Navier-Stokes system. Then as in other proofs of existence of strong solutions (see [5, 6, 16]), we study a linear system associated to our problem (Section 4):

$$
\mathbf{Z}^{\prime}=\boldsymbol{A} \mathbf{Z}+\mathbf{F} .
$$

We use a semi-group approach in this step as in [16] but we could also have used a Galerkin method as in [5]. Then we use the Banach fixed point theorem to prove the main result in Section 5. In order to do this, we need two technical results: estimates on $\mathbf{R}(\mathbf{Z})$ (Section 6) and estimates on the difference $\mathbf{R}^{1}\left(\mathbf{Z}^{1}\right)-\mathbf{R}^{2}\left(\mathbf{Z}^{2}\right)$ (Section 7$)$.

\section{Preliminaries and change of variables}

Let us first begin by introducing some notations and an important identity for the change of variables of surface integrals.

For a tensor $\boldsymbol{M}(\boldsymbol{x})=\left(m_{i j}(\boldsymbol{x})\right)_{i, j \in\{1,2,3\}}$ we use the notation

$$
[\operatorname{div} \boldsymbol{M}]_{i}=\sum_{j=1}^{3} \frac{\partial m_{i j}}{\partial x_{j}}
$$

and we denote by $\boldsymbol{M}^{t}$ the transpose of $\boldsymbol{M}, \operatorname{Cof} \boldsymbol{M}$ the cofactor matrix of the matrix $\boldsymbol{M}$ and recall that if $\boldsymbol{M}$ is invertible then

$$
\operatorname{Cof}(\boldsymbol{M})=(\operatorname{det} \boldsymbol{M}) \boldsymbol{M}^{-t}
$$

A deformation $\varphi$ is a smooth vector field

$$
\varphi: \bar{\Omega} \rightarrow \bar{\Omega}^{\varphi} \subset \mathbb{R}^{3}
$$

such that $\varphi: \Omega \rightarrow \Omega^{\varphi}$ is one-to-one, and orientation-preserving, i.e

$$
\operatorname{det} \nabla \boldsymbol{\varphi}(\boldsymbol{x})>0, \quad \forall \boldsymbol{x} \in \bar{\Omega} .
$$


Assume $\varphi$ is a deformation and assume $f: \Omega \rightarrow \mathbb{R}$ is an integrable function, then we have

$$
\int_{\partial \Omega^{\varphi}}\left(f \circ \varphi^{-1}\right) n^{\varphi} d \Gamma^{\varphi}=\int_{\partial \Omega}(\operatorname{det} \nabla \varphi) f(\nabla \varphi)^{-t} n d \Gamma,
$$

where $\boldsymbol{n}^{\boldsymbol{\varphi}}$ denotes the unit outer normal vector along the boundary of $\Omega^{\boldsymbol{\varphi}}$ and $\boldsymbol{n}$ is the outwardpointing unit normal vector along $\partial \Omega$ (see [3, p.40] for details).

Let us prove the existence a ball $B\left(\mathbf{0}, r_{1}\right)$ of $\mathbb{R}^{N_{0}}$ and a function $\phi: B\left(\mathbf{0}, r_{1}\right) \rightarrow \mathbb{R}$ such that for any function (regular enough) $\left(\alpha_{1}(t), \ldots, \alpha_{N_{0}}(t)\right) \in B\left(\mathbf{0}, r_{1}\right)$, any function $\boldsymbol{\xi}$ defined by (5) satisfies (3). More precisely, let us consider

$$
\xi(t, y):=\sum_{i=0}^{N_{0}} \alpha_{i}(t) \xi_{i}(y)
$$

we search $\alpha_{0}(t)=\phi\left(\alpha_{1}(t), \ldots, \alpha_{N_{0}}(t)\right)$ such that

$$
\begin{aligned}
\int_{\Omega_{S}} \operatorname{det}(\boldsymbol{\nabla} \varphi(t, \boldsymbol{y})) d \boldsymbol{y} & =\int_{\Omega_{S}} \operatorname{det}\left(\mathbf{I d}+\sum_{i=1}^{N_{0}} \alpha_{i}(t) \nabla \boldsymbol{\xi}_{i}(\boldsymbol{y})+\alpha_{0}(t) \nabla \boldsymbol{\xi}_{0}(\boldsymbol{y})\right) d \boldsymbol{y} \\
& =\mathcal{L}_{3}\left(\Omega_{S}\right)
\end{aligned}
$$

where $\mathcal{L}_{n}$ denotes the $\mathrm{n}$-dimensional Lebesgue measure $(n \in \mathbb{N})$. The above condition is equivalent to the fact that the deformation of the structure does not modify its volume which is compatible with the incompressibility of the fluid. Using the theorem of implicit functions, we can prove the following result.

LEMMA 2.1 There exist $r_{1}>0$ and $r_{2}>0$ and a mapping of class $C^{\infty}$

$$
\phi: B\left(\mathbf{0}, r_{1}\right) \subset \mathbb{R}^{N_{0}} \rightarrow B\left(0, r_{2}\right) \subset \mathbb{R},
$$

such that, for all $\left(\alpha_{1}, \ldots, \alpha_{N_{0}}\right) \in B\left(\mathbf{0}, r_{1}\right)$, there exists a unique $\alpha_{0}=\phi\left(\alpha_{1}, \ldots, \alpha_{N_{0}}\right) \in B\left(0, r_{2}\right)$ satisfying

$$
\int_{\Omega_{S}} \operatorname{det}\left(\mathbf{I d}+\sum_{i=1}^{N_{0}} \alpha_{i} \nabla \boldsymbol{\xi}_{i}(\boldsymbol{y})+\alpha_{0} \nabla \boldsymbol{\xi}_{0}(\boldsymbol{y})\right) d \boldsymbol{y}=\mathcal{L}_{3}\left(\Omega_{S}\right) .
$$

For the proof, see [1] Lemma 4.1 (see also [7] for a similar result). $\boldsymbol{\varphi}(t, \boldsymbol{y})=\boldsymbol{y}+\boldsymbol{\xi}(t, \boldsymbol{y})$. Let us remark that taking $r_{1}$ small enough, we also have $\varphi(t)$ and $\nabla \varphi(t)$ invertible.

REMARK 2.2 Let us remark that, from the fact that $\phi(\mathbf{0})=0$, and from the fact that $\phi$ is Lipschitz continuous, there exists a positive constant $\kappa$ such that

$$
\left|\alpha_{0}\right| \leqslant \kappa\|\boldsymbol{\alpha}\| .
$$

Here and in what follows, we write $\alpha:=\left(\alpha_{1}, \ldots, \alpha_{N_{0}}\right)$.

From (14) some calculations give

$$
\sum_{i=1}^{N_{0}} \varsigma_{i}(t) \beta_{i}(t)+\varsigma_{0}(t) \beta_{0}(t)=0
$$


with

$$
\beta_{i}(t)=\alpha_{i}^{\prime}(t)
$$

for any $0 \leqslant i \leqslant N_{0}$, and

$$
\begin{aligned}
\varsigma_{i}(t) & =\int_{\partial \Omega_{S}}(\operatorname{det} \nabla \boldsymbol{\varphi})(\nabla \boldsymbol{\varphi})^{-1} \xi_{i}(\boldsymbol{y}) \cdot \boldsymbol{n}_{\boldsymbol{y}} d \boldsymbol{\gamma}_{\boldsymbol{y}} \\
& =\int_{\partial \Omega_{S}(t)} \boldsymbol{\xi}_{i}\left(\boldsymbol{\varphi}^{-1}(t, \boldsymbol{x})\right) \cdot \boldsymbol{n}_{\boldsymbol{x}} d \boldsymbol{\gamma}_{\boldsymbol{x}}
\end{aligned}
$$

where the last equality is obtained thanks to (13).

Up to reducing the constant $r_{1}$ in Lemma 2.1, we can assume

$$
\|\operatorname{Cof}(\nabla \varphi)-\mathbf{I d}\|_{L^{\infty}\left(\partial \Omega_{S}\right)}<\frac{1}{2}
$$

and thus we can prove that

$$
\varsigma_{0}(t) \geqslant \frac{1}{2} \mathcal{L}_{2}\left(\partial \Omega_{S}(0)\right)>0 .
$$

Then we can define

$$
\widehat{\xi_{i}}(t, y)=\boldsymbol{\xi}_{i}(\boldsymbol{y})-\frac{\varsigma_{i}(t)}{\varsigma_{0}(t)} \boldsymbol{\xi}_{0}(\boldsymbol{y})
$$

which satisfies (from (16))

$$
\int_{\partial \Omega_{S}(t)} \widehat{\boldsymbol{\xi}_{i}}\left(t, \varphi^{-1}(t, \boldsymbol{x})\right) \cdot \boldsymbol{n}_{\boldsymbol{x}} d \boldsymbol{\gamma}_{\boldsymbol{x}}=0
$$

Then, (5) and (15) imply that the velocity of deformation is spanned by the family $\left\{\widehat{\boldsymbol{\xi}}_{1}, \ldots, \widehat{\boldsymbol{\xi}}_{N_{0}}\right\}$ :

$$
\frac{\partial \boldsymbol{\xi}}{\partial t}(t, \boldsymbol{y})=\sum_{i=1}^{N_{0}} \beta_{i}(t)\left(\boldsymbol{\xi}_{i}(\boldsymbol{y})-\frac{\varsigma_{i}(t)}{\varsigma_{0}(t)} \boldsymbol{\xi}_{0}(\boldsymbol{y})\right)=: \sum_{i=1}^{N_{0}} \beta_{i}(t) \widehat{\boldsymbol{\xi}}_{i}(t, \boldsymbol{y}) .
$$

REMARK 2.3 Let us remark that the family $\left\{\boldsymbol{\xi}_{i}\right\}_{i=0}^{N_{0}}$ and, for all $t$, the family $\left\{\widehat{\boldsymbol{\xi}}_{i}(t)\right\}_{i=1}^{N_{0}}$ defined as above, are linearly independent families.

In what follows, let us consider the elastic deformation $\varphi$

$$
\begin{aligned}
\varphi(t, \cdot): \Omega_{S} & \rightarrow \Omega_{S}(t) \\
\boldsymbol{y} & \mapsto \boldsymbol{y}+\boldsymbol{\xi}(t, \boldsymbol{y})
\end{aligned}
$$

associated with $\boldsymbol{\xi}$ which is the elastic displacement of the structure defined by (5) and which is the solution of (8). We will always assume that

$$
\left(\alpha_{1}(t), \ldots, \alpha_{N_{0}}(t)\right) \in B\left(\mathbf{0}, r_{1}\right),
$$

where $r_{1}$ is small enough to apply Lemma 2.1 and to have $\varphi(t)$ and $\nabla \varphi(t)$ invertible for all $t$ and (17). Recall that the latter condition implies (18). 
We construct a change of variables $\boldsymbol{X}: \Omega \rightarrow \Omega$ which transforms $\Omega_{S}$ onto $\Omega_{S}(t)$ and $\Omega_{F}$ onto $\Omega_{F}(t)$. We will use this change of variables to transform systems (7)-(8) into a system written in fixed domains. The idea is to extend the elastic deformation defined above on the domain of the fluid. Thus, we consider a linear extension operator $\varepsilon$ :

$$
\varepsilon: \boldsymbol{H}^{3}\left(\Omega_{S}\right) \rightarrow \boldsymbol{H}^{3}(\Omega) \cap \boldsymbol{H}_{0}^{1}(\Omega)
$$

such that for any $\boldsymbol{w} \in \boldsymbol{H}^{3}\left(\Omega_{S}\right)$ :

(i) $\varepsilon(\boldsymbol{w})=\boldsymbol{w}$ a.e in $\Omega_{S}$,

(ii) $\varepsilon(\boldsymbol{w})$ has support within $\left[\Omega_{S}\right]^{\epsilon}:=\left\{\boldsymbol{y} \in \Omega: \operatorname{dist}\left(\boldsymbol{y}, \Omega_{S}\right)<\epsilon\right\}$, with $0<\epsilon<\operatorname{dist}\left(\Omega_{S}, \partial \Omega\right)$,

(iii) $\|\varepsilon(\boldsymbol{w})\|_{\boldsymbol{H}^{3}(\Omega)} \leqslant C\|\boldsymbol{w}\|_{\boldsymbol{H}^{3}\left(\Omega_{S}\right)}$.

Using this operator, we can define an extension of $\varphi$ on $\Omega$ as follows: for each $t \in[0, T]$ we set

$$
\boldsymbol{X}(t, \boldsymbol{y}):=\boldsymbol{y}+\varepsilon(\varphi(t, y)-\boldsymbol{y})=\boldsymbol{y}+\varepsilon(\xi(t, y)), \forall \boldsymbol{y} \in \Omega .
$$

In what follows, we assume $\left(\alpha_{1}, \ldots, \alpha_{N_{0}}\right) \in\left[H^{2}(0, T)\right]^{N_{0}}$. Using the Sobolev embedding theorem, we will consider the following norms for $H^{2}(0, T)$ and $H^{1}(0, T)$ (which are equivalent to the usual norms):

$$
\|f\|_{\mathcal{H}^{2}(0, T)}:=\|f\|_{L^{\infty}(0, T)}+\left\|f^{\prime}\right\|_{L^{\infty}(0, T)}+\left\|f^{\prime \prime}\right\|_{L^{2}(0, T)}, \quad\left(f \in H^{2}(0, T)\right)
$$

and

$$
\|f\|_{\mathcal{H}^{1}(0, T)}:=\|f\|_{L^{\infty}(0, T)}+\left\|f^{\prime}\right\|_{L^{2}(0, T)}, \quad\left(f \in H^{1}(0, T)\right) .
$$

Let us note, that, since $\phi \in C^{\infty}\left(B\left(\mathbf{0}, r_{1}\right)\right)$, we have $\alpha_{0}=\phi\left(\alpha_{1}, \ldots, \alpha_{N_{0}}\right) \in H^{2}(0, T)$. Hence, we deduce that $\boldsymbol{\xi} \in \boldsymbol{H}^{2}\left(0, T ; \boldsymbol{H}^{3}\left(\Omega_{S}\right)\right)$ and by using Sobolev embedding theorems, we obtain

$$
\varepsilon(\xi) \in C^{1}\left([0, T] ; W^{1, \infty}(\Omega)\right)
$$

and

$$
\begin{aligned}
\|\mathcal{E}(\boldsymbol{\xi})\|_{\boldsymbol{L}^{\infty}\left(0, T ; \boldsymbol{W}^{1, \infty}(\Omega)\right)} & \leqslant C_{\mathcal{E}}\|\boldsymbol{\xi}\|_{\boldsymbol{L}^{\infty}\left(0, T ; \boldsymbol{W}^{1, \infty}\left(\Omega_{S}\right)\right)} \\
& \leqslant C_{\mathcal{E}} C_{S}\|\boldsymbol{\xi}\|_{\boldsymbol{L}^{\infty}\left(0, T ; \boldsymbol{H}^{3}\left(\Omega_{S}\right)\right)} \\
& \leqslant C_{\mathcal{E}} C_{S} C\|\boldsymbol{\alpha}\|_{\left[\boldsymbol{L}^{\infty}(0, T)\right]^{N_{0}}}
\end{aligned}
$$

From the above estimates, we deduce that if $\left(\alpha_{1}, \ldots, \alpha_{N_{0}}\right)$ satisfies for all $t$ the condition (22) with $r_{1}$ small enough (depending on the geometry and on the family $\left\{\xi_{i}\right\}$ ), then $\boldsymbol{X}(t, \cdot)$ is bijective from $\Omega$ onto $\Omega$.

Moreover its inverse $Y(t, \cdot): \Omega \rightarrow \Omega$ satisfies

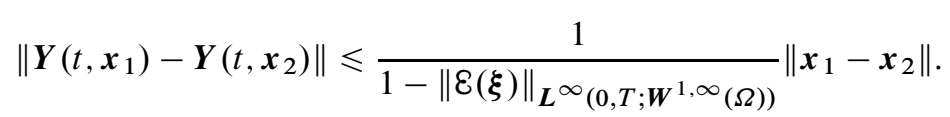

As a consequence, (see, for instance, [9, p.279]) $\boldsymbol{Y} \in \boldsymbol{L}^{\infty}\left(0, T ; \boldsymbol{W}^{1, \infty}(\Omega)\right)$. We can improve this regularity:

LEMMA 2.4 Assume $\alpha_{i} \in H^{2}(0, T), \boldsymbol{\xi}_{i} \in \boldsymbol{H}^{3}\left(\Omega_{S}\right)$ for all $i \in\left\{0,1, \ldots, N_{0}\right\}$ and assume (22) with $r_{1}$ small enough. Then $\boldsymbol{X} \in \boldsymbol{H}^{2}\left(0, T ; \boldsymbol{H}^{3}(\Omega)\right),(\nabla \boldsymbol{X})^{-1} \in \boldsymbol{H}^{2}\left(0, T ; \boldsymbol{H}^{2}(\Omega)\right)$ and

$$
\boldsymbol{Y} \in \boldsymbol{L}^{\infty}\left(0, T ; \boldsymbol{H}^{3}(\Omega)\right) \cap \boldsymbol{W}^{1, \infty}\left(0, T ; \boldsymbol{H}^{2}(\Omega)\right) \cap \boldsymbol{H}^{2}\left(0, T ; \boldsymbol{H}^{1}(\Omega)\right) .
$$


Proof. From (23) and from the regularity of $\alpha_{i}$ and $\boldsymbol{\xi}_{i}$, we have $\boldsymbol{X} \in \boldsymbol{H}^{2}\left(0, T ; \boldsymbol{H}^{3}(\Omega)\right)$.

Using the fact that $\boldsymbol{H}^{2}\left(0, T ; \boldsymbol{H}^{2}(\Omega)\right)$ is an algebra, we deduce that

$$
\operatorname{det} \nabla \boldsymbol{X} \in H^{2}\left(0, T ; H^{2}(\Omega)\right) \text { and } \operatorname{Cof}(\nabla \boldsymbol{X}) \in \boldsymbol{H}^{2}\left(0, T ; \boldsymbol{H}^{2}(\Omega)\right) .
$$

As $\boldsymbol{Y} \in \boldsymbol{L}^{\infty}\left(0, T ; \boldsymbol{W}^{1, \infty}(\Omega)\right)$, we have $\operatorname{det} \nabla \boldsymbol{X} \neq 0$ and thus the above equation implies

$$
\frac{1}{\operatorname{det} \nabla \boldsymbol{X}} \in H^{2}\left(0, T ; H^{2}(\Omega)\right) \text {. }
$$

Therefore, from (12) we have $(\nabla \boldsymbol{X})^{-1} \in \boldsymbol{H}^{2}\left(0, T ; \boldsymbol{H}^{2}(\Omega)\right)$. If we denote by $\boldsymbol{A}=(\nabla \boldsymbol{X})^{-1}$, we have $\boldsymbol{\nabla} \boldsymbol{Y}=\boldsymbol{A} \circ \boldsymbol{Y}$. Since $\boldsymbol{\nabla} \boldsymbol{Y} \in \boldsymbol{L}^{\infty}((0, T) \times \Omega)$,

$$
\begin{gathered}
\nabla^{2} \boldsymbol{Y}=(\nabla \boldsymbol{A})(\boldsymbol{Y})(\nabla \boldsymbol{Y}) \in \boldsymbol{L}^{\infty}\left(0, T ; \boldsymbol{L}^{6}(\Omega)\right) \\
\nabla^{3} \boldsymbol{Y}=\left(\nabla^{2} \boldsymbol{A}\right)(\boldsymbol{Y})(\boldsymbol{\nabla} \boldsymbol{Y})^{2}+(\boldsymbol{\nabla} \boldsymbol{A})(\boldsymbol{Y})\left(\nabla^{2} \boldsymbol{Y}\right) \in \boldsymbol{L}^{\infty}\left(0, T ; \boldsymbol{L}^{2}(\Omega)\right) .
\end{gathered}
$$

Hence,

$$
\boldsymbol{Y} \in \boldsymbol{L}^{\infty}\left(0, T ; \boldsymbol{H}^{3}(\Omega)\right) .
$$

Differentiating the identity $\boldsymbol{X}(t, \boldsymbol{Y}(t, \boldsymbol{x}))=\boldsymbol{x}$ with respect to time, we have

$$
\frac{\partial \boldsymbol{Y}}{\partial t}(t, \boldsymbol{x})=-\nabla \boldsymbol{Y}(t, \boldsymbol{x}) \frac{\partial \boldsymbol{X}}{\partial t}(t, \boldsymbol{Y}(t, \boldsymbol{x})) .
$$

Let us show that

$$
\boldsymbol{B}(t, \boldsymbol{x}):=\frac{\partial \boldsymbol{X}}{\partial t}(t, \boldsymbol{Y}(t, \boldsymbol{x})) \in \boldsymbol{L}^{\infty}\left(0, T ; \boldsymbol{H}^{2}(\Omega)\right) .
$$

From the regularity of $\boldsymbol{X}$, we already know that $\frac{\partial \boldsymbol{X}}{\partial t} \in \boldsymbol{C}\left([0, T] ; \boldsymbol{H}^{3}(\Omega)\right)$ and from the first part of the proof we have that $\boldsymbol{Y} \in \boldsymbol{L}^{\infty}\left(0, T ; \boldsymbol{H}^{3}(\Omega)\right)$. Differentiating (28) with respect to $\boldsymbol{x}$, we obtain

$$
\frac{\partial^{2} B_{i}}{\partial x_{k} \partial x_{j}}=\sum_{l, p=1}^{3} \frac{\partial^{3} X_{i}}{\partial t \partial y_{l} \partial y_{p}}(\boldsymbol{Y}) \frac{\partial Y_{p}}{\partial x_{k}} \frac{\partial Y_{l}}{\partial x_{j}}+\sum_{l=1}^{3} \frac{\partial^{2} X_{i}}{\partial t \partial y_{l}}(\boldsymbol{Y}) \frac{\partial^{2} Y_{l}}{\partial x_{j} \partial x_{k}} \in L^{\infty}\left(0, T ; L^{2}(\Omega)\right) .
$$

Therefore (28) holds true and, combined with (27), it implies

$$
\boldsymbol{Y} \in \boldsymbol{W}^{1, \infty}\left(0, T ; \boldsymbol{H}^{2}(\Omega)\right) .
$$

Differentiating (27) with respect to time, we have

$$
\frac{\partial^{2} \boldsymbol{Y}}{\partial t^{2}}=-\frac{\partial}{\partial t}(\nabla \boldsymbol{Y}) \frac{\partial \boldsymbol{X}}{\partial t}(\boldsymbol{Y})-\nabla \boldsymbol{Y}\left(\frac{\partial^{2} \boldsymbol{X}}{\partial t^{2}}(\boldsymbol{Y})+\frac{\partial(\boldsymbol{\nabla} \boldsymbol{X})}{\partial t}(\boldsymbol{Y}) \frac{\partial \boldsymbol{Y}}{\partial t}\right) \in \boldsymbol{L}^{2}\left(0, T ; \boldsymbol{H}^{1}(\Omega)\right) .
$$

Consequently,

$$
\boldsymbol{Y} \in \boldsymbol{H}^{2}\left(0, T ; \boldsymbol{H}^{1}(\Omega)\right) .
$$

This concludes the proof.

Let us also give the regularity of the $\widehat{\xi_{i}}$ defined by (19): 
LEMMA 2.5 Assume $\left\{\xi_{i}\right\}_{i=0}^{N_{0}} \subset \boldsymbol{H}^{3}\left(\Omega_{S}\right),\left\{\alpha_{i}\right\}_{i=0}^{N_{0}} \subset H^{2}(0, T)$ and (22) holds with $r_{1}$ small enough. Then

$$
\widehat{\boldsymbol{\xi}_{i}} \in \boldsymbol{H}^{2}\left(0, T ; \boldsymbol{H}^{3}\left(\Omega_{S}\right)\right) \text {. }
$$

Proof. From the regularity of $\boldsymbol{\xi}_{i}$ and $\alpha_{i}$ it follows that $\boldsymbol{\varphi} \in \boldsymbol{H}^{2}\left(0, T ; \boldsymbol{H}^{3}\left(\Omega_{S}\right)\right)$ and consequently, $\varsigma_{i} \in H^{2}(0, T)$. From (18), we deduce that $\frac{1}{\varsigma_{0}} \in H^{2}(0, T)$. This allows to deduce the desired regularity of $\widehat{\boldsymbol{\xi}}_{i}$.

\section{Writing the equations in fixed domains}

In this section, we use the change of variables constructed in Section 2 to write our system of equations (7)-(8) in fixed domains. We set

$$
\begin{gathered}
\boldsymbol{v}(t, \boldsymbol{y})=\operatorname{det}(\nabla \boldsymbol{X}(t, \boldsymbol{y}))(\nabla \boldsymbol{X}(t, \boldsymbol{y}))^{-1} \boldsymbol{u}(t, \boldsymbol{X}(t, \boldsymbol{y})) \\
q(t, \boldsymbol{y})=\operatorname{det}(\nabla \boldsymbol{X}(t, \boldsymbol{y})) p(t, \boldsymbol{X}(t, \boldsymbol{y})) \\
\boldsymbol{V}(t, \boldsymbol{y})=\operatorname{det}(\boldsymbol{\nabla} \boldsymbol{X}(t, \boldsymbol{y}))(\nabla \boldsymbol{X}(t, \boldsymbol{y}))^{-1} \frac{\partial \boldsymbol{\xi}}{\partial t}(t, \boldsymbol{y}) .
\end{gathered}
$$

Let us remark that we do not use the change of variables

$$
\boldsymbol{v}(t, y)=\boldsymbol{u}(t, X(t, y))
$$

because of the divergence equation in (7). More precisely we have the following result:

LEMMA 3.1 Let $v$ be defined by (29). Then

$$
(\operatorname{div} \boldsymbol{v})(t, \boldsymbol{y})=\operatorname{det} \boldsymbol{\nabla} \boldsymbol{X}(t, \boldsymbol{y})(\operatorname{div} \boldsymbol{u})(t, \boldsymbol{X}(t, \boldsymbol{y})) .
$$

Proof. To simplify the notation, we do not write in the proof the dependence in time of the variables. We have

$$
\begin{aligned}
\operatorname{div} \boldsymbol{v}(\boldsymbol{y})=\operatorname{div}[ & \operatorname{det}(\boldsymbol{\nabla} \boldsymbol{X}(\boldsymbol{y})) \boldsymbol{\nabla} \boldsymbol{Y}(\boldsymbol{X}(\boldsymbol{y})) \boldsymbol{u}(\boldsymbol{X}(\boldsymbol{y}))] \\
=\sum_{i, j=1}^{3} & {\left[\frac{\partial}{\partial y_{i}}(\operatorname{det} \boldsymbol{\nabla} \boldsymbol{X}(\boldsymbol{y})) \frac{\partial Y_{i}}{\partial x_{j}}(\boldsymbol{X}(\boldsymbol{y})) u_{j}(\boldsymbol{X}(\boldsymbol{y}))\right.} \\
& +\operatorname{det} \boldsymbol{\nabla} \boldsymbol{X}(\boldsymbol{y}) \sum_{l=1}^{3} \frac{\partial^{2} Y_{i}}{\partial x_{j} \partial x_{l}}(\boldsymbol{X}(\boldsymbol{y})) \frac{\partial X_{l}}{\partial y_{i}}(\boldsymbol{y}) u_{j}(\boldsymbol{X}(\boldsymbol{y})) \\
& \left.+\operatorname{det} \boldsymbol{\nabla} \boldsymbol{X}(\boldsymbol{y}) \sum_{l=1}^{3} \frac{\partial Y_{i}}{\partial x_{j}}(\boldsymbol{X}(\boldsymbol{y})) \frac{\partial u_{j}}{\partial x_{l}}(\boldsymbol{X}(\boldsymbol{y})) \frac{\partial X_{l}}{\partial y_{i}}(\boldsymbol{y})\right] .
\end{aligned}
$$

Using that

$$
\operatorname{det}(\nabla \boldsymbol{X})=\frac{1}{\operatorname{det}(\boldsymbol{\nabla} \boldsymbol{Y}(\boldsymbol{X}))}
$$


we have

$$
\begin{aligned}
\frac{\partial}{\partial y_{i}}(\operatorname{det} \nabla \boldsymbol{X}) & =-\frac{1}{(\operatorname{det} \nabla \boldsymbol{Y}(\boldsymbol{X}))^{2}} \frac{\partial}{\partial y_{i}}(\operatorname{det} \boldsymbol{\nabla}(\boldsymbol{X})) \\
& =-\frac{1}{(\operatorname{det} \nabla \boldsymbol{Y}(\boldsymbol{X}))^{2}}\left(\sum_{p, q=1}^{3} \operatorname{Cof}(\nabla \boldsymbol{Y}(\boldsymbol{X}))_{p q} \frac{\partial}{\partial y_{i}}(\nabla \boldsymbol{Y}(\boldsymbol{X}))_{p q}\right) \\
& =-\operatorname{det} \boldsymbol{\nabla} \boldsymbol{X}(\boldsymbol{y})\left(\sum_{p, q, l=1}^{3} \frac{\partial X_{q}}{\partial y_{p}}(\boldsymbol{y}) \frac{\partial^{2} Y_{p}}{\partial x_{q} \partial x_{l}}(\boldsymbol{X}(\boldsymbol{y})) \frac{\partial X_{l}}{\partial y_{i}}(\boldsymbol{y})\right) .
\end{aligned}
$$

Then

$$
\begin{aligned}
\operatorname{div} \boldsymbol{v}(\boldsymbol{y}) & =\operatorname{det} \boldsymbol{\nabla} \boldsymbol{X}(\boldsymbol{y}) \sum_{i, j, l=1}^{3} \frac{\partial Y_{i}}{\partial x_{j}}(\boldsymbol{X}(\boldsymbol{y})) \frac{\partial u_{j}}{\partial x_{l}}(\boldsymbol{X}(\boldsymbol{y})) \frac{\partial X_{l}}{\partial y_{i}}(\boldsymbol{y}) \\
& =\operatorname{det} \boldsymbol{\nabla} \boldsymbol{X}(\boldsymbol{y}) \sum_{j=1}^{3} \frac{\partial u_{j}}{\partial x_{j}}(\boldsymbol{X}(\boldsymbol{y}))=\operatorname{det}(\boldsymbol{\nabla} \boldsymbol{X}(\boldsymbol{y}))(\operatorname{div} \boldsymbol{u})(\boldsymbol{X}(\boldsymbol{y}))
\end{aligned}
$$

Now, we write the transformation of the derivative in time of $\boldsymbol{u}$. From (29) we have that for $i \in\{1,2,3\}$ :

$$
\begin{aligned}
\frac{\partial u_{i}}{\partial t}= & \sum_{k=1}^{3} \frac{\partial}{\partial t}\left((\operatorname{det} \nabla \boldsymbol{Y}) \frac{\partial X_{i}}{\partial y_{k}}(\boldsymbol{Y})\right) v_{k}(\boldsymbol{Y})+\sum_{k, l=1}^{3}(\operatorname{det} \nabla \boldsymbol{Y}) \frac{\partial X_{i}}{\partial y_{k}}(\boldsymbol{Y}) \frac{\partial v_{k}}{\partial y_{l}}(\boldsymbol{Y}) \frac{\partial Y_{l}}{\partial t} \\
& +(\operatorname{det} \nabla \boldsymbol{Y}) \frac{\partial v_{i}}{\partial t}(\boldsymbol{Y})+\sum_{k=1}^{3} \operatorname{det} \nabla \boldsymbol{Y}\left(\frac{\partial X_{i}}{\partial y_{k}}(\boldsymbol{Y})-\delta_{i, k}\right) \frac{\partial v_{k}}{\partial t}(\boldsymbol{Y}) .
\end{aligned}
$$

We calculate the transformation of the gradient of $\boldsymbol{u}$. As in the proof of Lemma 3.1, we do not write the dependence in time of the variables.

$$
\frac{\partial u_{i}}{\partial x_{j}}=(\operatorname{det} \nabla \boldsymbol{Y}) \frac{\partial v_{i}}{\partial y_{j}}(\boldsymbol{Y})+E_{i j}[\boldsymbol{v}]
$$

with,

$$
\begin{aligned}
E_{i j}[\boldsymbol{v}]= & \sum_{k=1}^{3}\left[\frac{\partial}{\partial x_{j}}\left((\operatorname{det} \nabla \boldsymbol{Y}) \frac{\partial X_{i}}{\partial y_{k}}(\boldsymbol{Y})\right) v_{k}(\boldsymbol{Y})+\operatorname{det} \nabla \boldsymbol{Y}\left(\frac{\partial X_{i}}{\partial y_{k}}(\boldsymbol{Y})-\delta_{i, k}\right) \frac{\partial v_{k}}{\partial y_{j}}(\boldsymbol{Y})\right. \\
& \left.+(\operatorname{det} \nabla \boldsymbol{Y}) \frac{\partial X_{i}}{\partial y_{k}}(\boldsymbol{Y}) \sum_{l=1}^{3} \frac{\partial v_{k}}{\partial y_{l}}(\boldsymbol{Y})\left((\nabla \boldsymbol{X})_{l, j}^{-1}(\boldsymbol{Y})-\delta_{l, j}\right)\right] .
\end{aligned}
$$


Then,

$$
\begin{aligned}
\frac{\partial^{2} u_{i}}{\partial x_{j}^{2}}= & (\operatorname{det} \nabla \boldsymbol{Y}) \frac{\partial^{2} v_{i}}{\partial y_{j}^{2}}(\boldsymbol{Y})+(\operatorname{det} \nabla \boldsymbol{Y}) \sum_{l=1}^{3} \frac{\partial^{2} v_{i}}{\partial y_{j} \partial y_{l}}(\boldsymbol{Y})\left((\nabla \boldsymbol{X})_{l, j}^{-1}(\boldsymbol{Y})-\delta_{l, j}\right) \\
& +\frac{\partial}{\partial x_{j}}(\operatorname{det} \nabla \boldsymbol{Y}) \frac{\partial v_{i}}{\partial y_{j}}(\boldsymbol{Y})+\frac{\partial}{\partial x_{j}}\left(E_{i j}[\boldsymbol{v}]\right) .
\end{aligned}
$$

For the nonlinear term we have:

$$
\begin{aligned}
{[(\boldsymbol{u} \cdot \nabla) \boldsymbol{u}]_{i}=} & \sum_{j=1}^{3}(\operatorname{det} \nabla \boldsymbol{Y})^{2} \frac{\partial v_{i}}{\partial y_{j}}(\boldsymbol{Y}) \sum_{m=1}^{3} \frac{\partial X_{j}}{\partial y_{m}}(\boldsymbol{Y}) v_{m}(\boldsymbol{Y}) \\
& +\sum_{j=1}^{3}(\operatorname{det} \nabla \boldsymbol{Y}) E_{i j}[\boldsymbol{v}] \sum_{m=1}^{3} \frac{\partial X_{j}}{\partial y_{m}}(\boldsymbol{Y}) v_{m}(\boldsymbol{Y})
\end{aligned}
$$

Finally, from (30) we have:

$$
\begin{aligned}
\frac{\partial p}{\partial x_{i}}= & \frac{\partial}{\partial x_{i}}(\operatorname{det} \nabla \boldsymbol{Y}) q(\boldsymbol{Y})+(\operatorname{det} \nabla \boldsymbol{Y}) \frac{\partial q}{\partial y_{i}}(\boldsymbol{Y}) \\
& +(\operatorname{det} \nabla \boldsymbol{Y}) \sum_{l=1}^{3} \frac{\partial q}{\partial y_{l}}(\boldsymbol{Y})\left((\boldsymbol{\nabla} \boldsymbol{X})_{l, i}^{-1}(\boldsymbol{Y})-\delta_{l, i}\right) .
\end{aligned}
$$

Thereby, we can rewrite (7) as follows

$$
\left\{\begin{array}{lll}
\frac{\partial \boldsymbol{v}}{\partial t}+[\boldsymbol{M} \boldsymbol{v}]-v[\boldsymbol{L} \boldsymbol{v}]+[\boldsymbol{N} \boldsymbol{v}]+[\boldsymbol{G} q]=\mathbf{0} & \text { in } & \Omega_{F}, t \in(0, T), \\
\operatorname{div}(\boldsymbol{v})=0 & \text { in } & \Omega_{F}, t \in(0, T), \\
\boldsymbol{v}=\mathbf{0} & \text { on } & \partial \Omega_{t} t \in(0, T), \\
\boldsymbol{v}=\boldsymbol{V} & \text { on } & \partial \Omega_{S}, t \in(0, T),
\end{array}\right.
$$

where $[\boldsymbol{L} \boldsymbol{v}],[\boldsymbol{M} \boldsymbol{v}],[\boldsymbol{N} \boldsymbol{v}],[\boldsymbol{G} q]$, are defined by

$$
\begin{aligned}
{[\boldsymbol{M} \boldsymbol{v}]_{i}:=} & \operatorname{det} \nabla \boldsymbol{X} \sum_{k=1}^{3} \frac{\partial}{\partial t}\left(\operatorname{det} \nabla \boldsymbol{Y} \frac{\partial X_{i}}{\partial y_{k}}(\boldsymbol{Y})\right)(\boldsymbol{X}) v_{k}+\sum_{k, l=1}^{3} \frac{\partial X_{i}}{\partial y_{k}} \frac{\partial v_{k}}{\partial y_{l}} \frac{\partial Y_{l}}{\partial t}(\boldsymbol{X}) \\
& +\sum_{k=1}^{3}\left(\frac{\partial X_{i}}{\partial y_{k}}-\delta_{i, k}\right) \frac{\partial v_{k}}{\partial t}, \\
{[\boldsymbol{L} \boldsymbol{v}]_{i}:=} & \sum_{j=1}^{3}\left[\frac{\partial^{2} v_{i}}{\partial y_{j}^{2}}+\sum_{l=1}^{3} \frac{\partial^{2} v_{i}}{\partial y_{j} \partial y_{l}}\left((\nabla \boldsymbol{X})_{l, j}^{-1}-\delta_{l, j}\right)\right. \\
& \left.+(\operatorname{det} \nabla \boldsymbol{X}) \frac{\partial}{\partial x_{j}}(\operatorname{det} \nabla \boldsymbol{Y})(\boldsymbol{X}) \frac{\partial v_{i}}{\partial y_{j}}+(\operatorname{det} \nabla \boldsymbol{X}) \frac{\partial}{\partial x_{j}}\left(E_{i j}[\boldsymbol{v}]\right)(\boldsymbol{X})\right], \\
{[\boldsymbol{N} \boldsymbol{v}]_{i}:=} & \frac{1}{\operatorname{det} \boldsymbol{\nabla} \boldsymbol{X}} \sum_{j=1} \frac{\partial v_{i}}{\partial y_{j}} \sum_{m=1}^{3} \frac{\partial X_{j}}{\partial y_{m}} v_{m}+\sum_{j=1}^{3} E_{i j}[\boldsymbol{v}](\boldsymbol{X}) \sum_{m} \frac{\partial X_{j}}{\partial y_{m}} v_{m},
\end{aligned}
$$




$$
[\boldsymbol{G} q]_{i}:=(\operatorname{det} \nabla \boldsymbol{X}) \frac{\partial}{\partial x_{i}}(\operatorname{det} \nabla \boldsymbol{Y})(\boldsymbol{X}) q+\frac{\partial q}{\partial y_{i}}+\sum_{l=1}^{3} \frac{\partial q}{\partial y_{l}}\left((\nabla \boldsymbol{X})_{l, i}^{-1}-\delta_{l, i}\right) .
$$

From (31) and thanks to (21) and (12), $\boldsymbol{V}$ can be written as

$$
\boldsymbol{V}(t, \boldsymbol{y})=\sum_{i=1}^{N_{0}} \beta_{i}(t) \widehat{\boldsymbol{\eta}}_{i}(t, \boldsymbol{y}):=\sum_{i=1}^{N_{0}} \beta_{i} \xi_{i}+[\boldsymbol{H} \boldsymbol{\beta}]
$$

where

$$
\widehat{\boldsymbol{\eta}}_{i}(t, \boldsymbol{y})=(\operatorname{Cof} \nabla \boldsymbol{X})^{t} \widehat{\xi}_{i}(t, \boldsymbol{y}) \quad \text { and } \quad[\boldsymbol{H} \boldsymbol{\beta}]=\sum_{i=1}^{N_{0}} \beta_{i}\left(\widehat{\boldsymbol{\eta}}_{i}-\boldsymbol{\xi}_{i}\right) .
$$

Using (4), (13) and (20) we can prove that the function $[\boldsymbol{H} \boldsymbol{\beta}]$ satisfies

$$
\int_{\partial \Omega_{S}}[\boldsymbol{H} \boldsymbol{\beta}] \cdot \boldsymbol{n}=0 .
$$

Consequently, for each $i=1,2, \ldots, N_{0}$, we can consider the following Stokes problem

$$
\left\{\begin{array}{lll}
-v \boldsymbol{\Delta} \boldsymbol{W}_{i}+\nabla \pi_{i}=\mathbf{0} & \text { in } & \Omega_{F} \\
\operatorname{div}\left(\boldsymbol{W}_{i}\right)=0 & \text { in } & \Omega_{F} \\
\boldsymbol{W}_{i}=\mathbf{0} & \text { on } & \partial \Omega \\
\boldsymbol{W}_{i}=\widehat{\boldsymbol{\eta}}_{i}-\boldsymbol{\xi}_{i} & \text { on } & \partial \Omega_{S}
\end{array}\right.
$$

and we can write

$$
\boldsymbol{w}=\boldsymbol{v}-\sum_{i=1}^{N_{0}} \beta_{i} \boldsymbol{W}_{i}
$$

and

$$
\pi=q-\sum_{i=1}^{N_{0}} \beta_{i} \pi_{i}
$$

Then, from (38), we deduce that $(\boldsymbol{w}, \pi)$ satisfies

$$
\left\{\begin{array}{lll}
\frac{\partial \boldsymbol{w}}{\partial t}+[\boldsymbol{M} \boldsymbol{w}]-v[\boldsymbol{L} \boldsymbol{w}]+[\boldsymbol{G} \pi]=\boldsymbol{K} & \text { in } & \Omega_{F}, t \in(0, T) \\
\operatorname{div}(\boldsymbol{w})=0 & \text { in } & \Omega_{F}, t \in(0, T) \\
\boldsymbol{w}=\mathbf{0} & \text { on } & \partial \Omega, t \in(0, T) \\
\boldsymbol{w}=\sum_{i=1}^{N_{0}} \beta_{i} \boldsymbol{\xi}_{i} & \text { on } & \partial \Omega_{S}, t \in(0, T)
\end{array}\right.
$$

with

$$
\begin{aligned}
\boldsymbol{K}=-\sum_{i=1}^{N_{0}} \beta_{i}^{\prime} \boldsymbol{W}_{i}-\sum_{i=1}^{N_{0}} \beta_{i} \boldsymbol{W}_{i}^{\prime}-\sum_{i=1}^{N_{0}}\left[\boldsymbol{M}\left(\beta_{i} \boldsymbol{W}_{i}\right)\right]+v \sum_{i=1}^{N_{0}}\left[\boldsymbol{L}\left(\beta_{i} \boldsymbol{W}_{i}\right)\right] \\
-\left[\boldsymbol{N}\left(\boldsymbol{w}+\sum_{i=1}^{N_{0}} \beta_{i} \boldsymbol{W}_{i}\right)\right]-\sum_{i=1}^{N_{0}}\left[\boldsymbol{G}\left(\beta_{i} \pi_{i}\right)\right] .
\end{aligned}
$$


Now, we transform also the equation for the structure in order to have fixed domains in the integrals coming from the fluid and in order to decompose the elastic displacement only by using $\boldsymbol{\xi}_{i}, i=$ $1, \ldots, N_{0}$.

From (6) we have

$$
\begin{aligned}
\int_{\Omega_{S}} \frac{\partial^{2} \boldsymbol{\xi}}{\partial t^{2}} \cdot \widehat{\boldsymbol{\xi}}_{i} d \boldsymbol{y}= & \sum_{j=1}^{N_{0}} \beta_{j}^{\prime} \int_{\Omega_{S}} \boldsymbol{\xi}_{j} \cdot \boldsymbol{\xi}_{i} d \boldsymbol{y}+\sum_{j=1}^{N_{0}} \beta_{j}^{\prime} \int_{\Omega_{S}}\left(\widehat{\boldsymbol{\xi}}_{j}-\boldsymbol{\xi}_{j}\right) \cdot \boldsymbol{\xi}_{i} d \boldsymbol{y} \\
& +\sum_{j=1}^{N_{0}} \beta_{j} \int_{\Omega_{S}} \frac{\partial}{\partial t}\left(\widehat{\boldsymbol{\xi}}_{j}\right) \cdot \widehat{\boldsymbol{\xi}}_{i} d \boldsymbol{y}+\sum_{j=1}^{N_{0}} \beta_{j}^{\prime} \int_{\Omega_{S}} \widehat{\boldsymbol{\xi}}_{j} \cdot\left(\widehat{\boldsymbol{\xi}}_{i}-\boldsymbol{\xi}_{i}\right) d \boldsymbol{y} .
\end{aligned}
$$

On the other hand, from (13), (33) and (46)

$$
\begin{array}{r}
\int_{\partial \Omega_{S}(t)} \boldsymbol{\sigma}(\boldsymbol{u}, p) \boldsymbol{n}_{\boldsymbol{x}} \cdot \widehat{\boldsymbol{\xi}}_{i}(\boldsymbol{Y}) d \boldsymbol{\gamma}_{\boldsymbol{x}}=\int_{\partial \Omega_{S}} \boldsymbol{\sigma}(\boldsymbol{w}, \pi) \boldsymbol{n}_{\boldsymbol{y}} \cdot \boldsymbol{\xi}_{i} d \boldsymbol{\gamma}_{\boldsymbol{y}}+\int_{\partial \Omega_{S}} \boldsymbol{\sigma}(\boldsymbol{w}, \pi) \boldsymbol{n}_{\boldsymbol{y}} \cdot\left(\widehat{\boldsymbol{\xi}}_{i}-\boldsymbol{\xi}_{i}\right) d \boldsymbol{\gamma}_{\boldsymbol{y}} \\
+\int_{\partial \Omega_{S}} \boldsymbol{G}_{0}[\boldsymbol{w}, \pi] \cdot \widehat{\boldsymbol{\xi}}_{i} d \boldsymbol{\gamma}_{\boldsymbol{y}}
\end{array}
$$

with

$$
\begin{array}{r}
\boldsymbol{G}_{0}[\boldsymbol{w}, \pi]=\boldsymbol{\sigma}(\boldsymbol{w}, \pi)\left((\boldsymbol{\nabla})^{-t}-\mathbf{I d}\right) \boldsymbol{n}_{\boldsymbol{y}}+\sum_{i=1}^{N_{0}} \beta_{i} \boldsymbol{\sigma}\left(\boldsymbol{W}_{i}, \pi_{i}\right)(\nabla \boldsymbol{X})^{-t} \boldsymbol{n}_{\boldsymbol{y}} \\
+2 v\left(\boldsymbol{E}[\boldsymbol{w}]+(\boldsymbol{E}[\boldsymbol{w}])^{t}\right)(\boldsymbol{X}) \operatorname{Cof}(\nabla \boldsymbol{X}) \boldsymbol{n}_{\boldsymbol{y}}+2 v \sum_{i=1}^{N_{0}} \beta_{i}\left(\boldsymbol{E}\left[\boldsymbol{W}_{i}\right]\right. \\
\left.+\left(\boldsymbol{E}\left[\boldsymbol{W}_{i}\right]\right)^{t}\right)(\boldsymbol{X}) \operatorname{Cof}(\nabla \boldsymbol{X}) \boldsymbol{n}_{\boldsymbol{y}}
\end{array}
$$

and with $\boldsymbol{E}[\boldsymbol{w}]$ defined by (34).

Thus, we can write (8) as follows

$$
\sum_{j=1}^{N_{0}} \beta_{j}^{\prime} \int_{\Omega_{S}} \xi_{j} \cdot \xi_{i} d \boldsymbol{y}=\int_{\partial \Omega_{S}} \boldsymbol{\sigma}(\boldsymbol{w}, \pi) \boldsymbol{n}_{\boldsymbol{y}} \cdot \boldsymbol{\xi}_{i} d \boldsymbol{\gamma}_{\boldsymbol{y}}+f_{i},
$$

where $\boldsymbol{f} \in\left[L^{2}(0, T)\right]^{N_{0}}$ is given by

$$
\begin{aligned}
f_{i}= & -\sum_{j=1}^{N_{0}} \beta_{j}^{\prime} \int_{\Omega_{S}}\left(\widehat{\boldsymbol{\xi}}_{j}-\boldsymbol{\xi}_{j}\right) \cdot \boldsymbol{\xi}_{i} d \boldsymbol{y}-\sum_{j=1}^{N_{0}} \beta_{j} \int_{\Omega_{S}} \frac{\partial}{\partial t}\left(\widehat{\boldsymbol{\xi}}_{j}\right) \cdot \widehat{\boldsymbol{\xi}}_{i} d \boldsymbol{y} \\
& -\sum_{j=1}^{N_{0}} \beta_{j}^{\prime} \int_{\Omega_{S}} \widehat{\boldsymbol{\xi}}_{j} \cdot\left(\widehat{\boldsymbol{\xi}}_{i}-\boldsymbol{\xi}_{i}\right) d \boldsymbol{y}-2 \mu \int_{\Omega_{S}} \boldsymbol{\varepsilon}(\boldsymbol{\xi}): \boldsymbol{\varepsilon}\left(\widehat{\boldsymbol{\xi}}_{i}\right) d \boldsymbol{y}-\lambda \int_{\Omega_{S}} \operatorname{div}(\boldsymbol{\xi}) \operatorname{div}\left(\widehat{\boldsymbol{\xi}}_{i}\right) d \boldsymbol{y} \\
& +\int_{\partial \Omega_{S}} \boldsymbol{\sigma}(\boldsymbol{w}, \pi) \boldsymbol{n}_{\boldsymbol{y}} \cdot\left(\widehat{\boldsymbol{\xi}}_{i}-\boldsymbol{\xi}_{i}\right) d \boldsymbol{\gamma}_{\boldsymbol{y}}+\int_{\partial \Omega_{S}} \boldsymbol{G}_{0}[\boldsymbol{w}, \pi] \cdot \widehat{\boldsymbol{\xi}}_{i} d \boldsymbol{\gamma}_{\boldsymbol{y}}, \quad i \in\left\{1,2, \ldots, N_{0}\right\} .
\end{aligned}
$$

Then we have the following proposition: 
Proposition 3.2 Assume $(\boldsymbol{w}, \pi)$ is defined by (29)-(30) and (46)-(47). Then

$$
(\boldsymbol{u}, p, \boldsymbol{\beta}) \in \Phi(t) \times L^{2}\left(0, T ; H^{1}\left(\Omega_{F}(t)\right)\right) \times\left[H^{1}(0, T)\right]^{N_{0}}
$$

if and only if

$$
(\boldsymbol{w}, \pi, \boldsymbol{\beta}) \in \Phi \times L^{2}\left(0, T ; H^{1}\left(\Omega_{F}\right)\right) \times\left[H^{1}(0, T)\right]^{N_{0}} .
$$

Moreover, if $(\boldsymbol{u}, p, \boldsymbol{\beta})$ satisfies (53) then $(\boldsymbol{u}, p, \boldsymbol{\beta})$ is solution of (7)-(8) along with the initial conditions (9) if and only if ( $\boldsymbol{w}, \boldsymbol{\pi}, \boldsymbol{\beta})$ is solution of (48), (51) with (39)-(42), (49), (50) and (52) and with the initial conditions

$$
\boldsymbol{w}_{\left.\right|_{t=0}}=\boldsymbol{w}^{0}=\boldsymbol{u}^{0}-\sum_{i=1}^{N_{0}} \beta_{i}^{0} \boldsymbol{W}_{i} \quad \text { in } \quad \Omega_{F}
$$

and $\beta_{i}^{0}=\beta_{i}(0)$.

In the remaining part of the article, we study the problem (48), (51). To prove the local in time existence of such a system we use a similar approach as in [16]. More precisely we write (48), (51) as

$$
\left\{\begin{array}{lll}
\frac{\partial \boldsymbol{w}}{\partial t}-v \boldsymbol{\Delta} \boldsymbol{w}+\nabla \pi=\boldsymbol{F} & \text { in } & \Omega_{F}, t \in(0, T) \\
\operatorname{div}(\boldsymbol{w})=0 & \text { in } & \Omega_{F}, t \in(0, T) \\
\boldsymbol{w}=\mathbf{0} & \text { on } & \partial \Omega, t \in(0, T) \\
\boldsymbol{w}=\sum_{i=1}^{N_{0}} \beta_{i} \xi_{i} & \text { on } & \partial \Omega_{S}, t \in(0, T)
\end{array}\right.
$$

and

$$
[\mathbb{A} \boldsymbol{\beta}]_{i}^{\prime}=\int_{\partial \Omega_{S}} \boldsymbol{\sigma}(\boldsymbol{w}, \pi) \boldsymbol{n}_{\boldsymbol{y}} \cdot \boldsymbol{\xi}_{i} d \boldsymbol{\gamma}_{\boldsymbol{y}}+f_{i}
$$

with $\mathbb{A}$ defined by

$$
(\mathbb{A})_{i, j}=\int_{\Omega_{S}} \boldsymbol{\xi}_{i} \cdot \boldsymbol{\xi}_{j} d \boldsymbol{y},
$$

with $f_{i}$ given by (52) and with

$$
\boldsymbol{F}=\boldsymbol{K}-[\boldsymbol{M} \boldsymbol{w}]+v[(\boldsymbol{L}-\boldsymbol{\Delta}) \boldsymbol{w}]+[(\boldsymbol{\nabla}-\boldsymbol{G}) \pi] .
$$

\section{Linear problem associated to (48), (51)}

In this section, we consider the following linear problem

$$
\left\{\begin{array}{lll}
\frac{\partial \tilde{\boldsymbol{w}}}{\partial t}-v \boldsymbol{\Delta} \tilde{\boldsymbol{w}}+\nabla \tilde{\pi}=\boldsymbol{F} & \text { in } & \Omega_{F}, t \in(0, T) \\
\operatorname{div}(\tilde{\boldsymbol{w}})=0 & \text { in } & \Omega_{F}, t \in(0, T) \\
\tilde{\boldsymbol{w}}=\mathbf{0} & \text { on } & \partial \Omega_{t} t \in(0, T) \\
\tilde{\boldsymbol{w}}=\sum_{i=1}^{N_{0}} \widetilde{\beta}_{i} \boldsymbol{\xi}_{i} & \text { on } & \partial \Omega_{S}, t \in(0, T)
\end{array}\right.
$$

and

$$
[\mathbb{A} \widetilde{\boldsymbol{\beta}}]_{j}^{\prime}=\int_{\partial \Omega_{S}} \boldsymbol{\sigma}(\widetilde{\boldsymbol{w}}, \widetilde{\pi}) n_{\boldsymbol{y}} \cdot \boldsymbol{\xi}_{j} d \boldsymbol{\gamma}_{\boldsymbol{y}}+f_{j}
$$


along with the initial conditions

$$
\widetilde{\boldsymbol{w}}(0)=\boldsymbol{w}^{0}, \quad \text { and } \widetilde{\boldsymbol{\beta}}(0)=\boldsymbol{\beta}^{0},
$$

where $\boldsymbol{F}, f_{j}$ are given functions, and $\beta_{j}^{0}$ are given real numbers. Recall that $\mathbb{A}$ is defined by (56).

In order to study (58)-(59), we use a semigroup approach. More precisely, let us set

$$
\begin{gathered}
\boldsymbol{H}=\left\{(\boldsymbol{w}, \boldsymbol{\beta}) \in \boldsymbol{L}^{2}\left(\Omega_{F}\right) \times \mathbb{R}^{N_{0}}: \operatorname{div}(\boldsymbol{w})=0 \text { in } \Omega_{F}\right. \\
\boldsymbol{w} \cdot \boldsymbol{n}=\mathbf{0} \text { on } \quad \partial \Omega \\
\left.\boldsymbol{w} \cdot \boldsymbol{n}=\left(\sum_{i=1}^{N_{0}} \beta_{i} \boldsymbol{\xi}_{i}\right) \cdot \boldsymbol{n} \text { on } \partial \Omega_{S}\right\}, \\
D(\boldsymbol{A})=\left\{\begin{array}{r}
(\boldsymbol{w}, \boldsymbol{\beta}) \in \boldsymbol{H}: \boldsymbol{w} \in \boldsymbol{H}^{2}\left(\Omega_{F}\right) \\
\boldsymbol{w}=\mathbf{0} \text { on } \partial \Omega \\
\left.\boldsymbol{w}=\sum_{i=1}^{N_{0}} \beta_{i} \boldsymbol{\xi}_{i} \text { on } \partial \Omega_{S}\right\}, \\
Q: D(\boldsymbol{A}) \rightarrow \boldsymbol{L}^{2}\left(\Omega_{F}\right) \times \mathbb{R}^{N_{0}} \\
(\boldsymbol{w}, \boldsymbol{\beta}) \mapsto\left(-v \boldsymbol{\Delta} \boldsymbol{w},\left(\mathbb{A}^{-1} \mathbb{B}(\boldsymbol{w})\right)\right)
\end{array}\right.
\end{gathered}
$$

and

$$
\begin{aligned}
\boldsymbol{A}: D(\boldsymbol{A}) & \rightarrow \boldsymbol{H} \\
(\boldsymbol{w}, \boldsymbol{\beta}) & \mapsto \mathbb{P}(Q(\boldsymbol{w}, \boldsymbol{\beta}))
\end{aligned}
$$

where $\mathbb{B}_{j}(\boldsymbol{w})=\int_{\partial \Omega_{S}} 2 v \boldsymbol{\varepsilon}(\boldsymbol{w}) \boldsymbol{n} \cdot \boldsymbol{\xi}_{j} d \boldsymbol{\gamma}_{\boldsymbol{y}}$ and $\mathbb{P}: \boldsymbol{L}^{2}\left(\Omega_{F}\right) \times \mathbb{R}^{N_{0}} \rightarrow \boldsymbol{H}$ is the orthogonal projection.

Here we have used the following scalar product for $\boldsymbol{L}^{2}\left(\Omega_{F}\right) \times \mathbb{R}^{N_{0}}$ :

$$
((\boldsymbol{w}, \boldsymbol{\beta}),(\boldsymbol{u}, \boldsymbol{\alpha})):=\int_{\Omega_{F}} \boldsymbol{w} \cdot \boldsymbol{u} d \boldsymbol{y}+\mathbb{A} \boldsymbol{\beta} \cdot \boldsymbol{\alpha} .
$$

Its associated norm is equivalent to the usual one. Let us also remark that $\boldsymbol{H}$ is a closed subspace of the Hilbert space $\boldsymbol{L}^{2}\left(\Omega_{F}\right) \times \mathbb{R}^{N_{0}}$.

To study the operator $\boldsymbol{A}$, we also need the following function space

$$
\begin{aligned}
\boldsymbol{V}=\{(\boldsymbol{w}, \boldsymbol{\beta}) \in \boldsymbol{H}: \boldsymbol{w} & \in \boldsymbol{H}^{1}\left(\Omega_{F}\right) \\
\boldsymbol{w} & =\mathbf{0} \text { on } \partial \Omega \\
\boldsymbol{w} & \left.=\sum_{i=1}^{N_{0}} \beta_{i} \xi_{i} \text { on } \partial \Omega_{S}\right\} .
\end{aligned}
$$

Proposition 4.1 The operator $\boldsymbol{A}: D(\boldsymbol{A}) \rightarrow \boldsymbol{H}$ is positive and self-adjoint. In particular, $-\boldsymbol{A}$ is the generator of a contraction semigroup on $\boldsymbol{H}$. 
Proof. Assume $(\boldsymbol{u}, \boldsymbol{\alpha}),(\boldsymbol{w}, \boldsymbol{\beta}) \in D(\boldsymbol{A})$. Then

$$
\begin{aligned}
(\boldsymbol{A}(\boldsymbol{w}, \boldsymbol{\beta}),(\boldsymbol{u}, \boldsymbol{\alpha}))= & (\mathbb{Q}(\boldsymbol{w}, \boldsymbol{\beta}),(\boldsymbol{u}, \boldsymbol{\alpha})) \\
= & \int_{\Omega_{F}}-v \boldsymbol{\Delta} \boldsymbol{w} \cdot \boldsymbol{u} d \boldsymbol{y}+\mathbb{A A}^{-1} \mathbb{B}(\boldsymbol{w}) \cdot \boldsymbol{\alpha} \\
= & 2 v \int_{\Omega_{F}} \boldsymbol{\varepsilon}(\boldsymbol{w}): \boldsymbol{\varepsilon}(\boldsymbol{u}) d \boldsymbol{y}-2 v \int_{\partial \Omega_{S}} \boldsymbol{\varepsilon}(\boldsymbol{w}) \boldsymbol{n} \cdot \boldsymbol{u} d \boldsymbol{\gamma}_{\boldsymbol{y}} \\
& \quad+\sum_{j=1}^{N_{0}} 2 v \int_{\partial \Omega_{S}} \boldsymbol{\varepsilon}(\boldsymbol{w}) \boldsymbol{n} \cdot \boldsymbol{\xi}_{j} d \boldsymbol{\gamma}_{\boldsymbol{y}} \alpha_{j} \\
& =2 v \int_{\Omega_{F}} \boldsymbol{\varepsilon}(\boldsymbol{w}): \boldsymbol{\varepsilon}(\boldsymbol{u}) d \boldsymbol{y}
\end{aligned}
$$

Thus

$$
(\boldsymbol{A}(\boldsymbol{w}, \boldsymbol{\beta}),(\boldsymbol{u}, \boldsymbol{\alpha}))=2 v \int_{\Omega_{F}} \boldsymbol{\varepsilon}(\boldsymbol{w}): \boldsymbol{\varepsilon}(\boldsymbol{u}) d \boldsymbol{y}, \quad \forall(\boldsymbol{u}, \boldsymbol{\alpha}),(\boldsymbol{w}, \boldsymbol{\beta}) \in D(\boldsymbol{A}) .
$$

In particular, $\boldsymbol{A}$ is symmetric. Moreover, $\mathbf{I d}+\boldsymbol{A}$ is onto: let us take $(\boldsymbol{v}, \boldsymbol{\gamma}) \in \boldsymbol{H}$. The equation

$$
(\mathbf{I d}+\boldsymbol{A})(\boldsymbol{w}, \boldsymbol{\beta})=(\boldsymbol{v}, \boldsymbol{\gamma})
$$

is equivalent to

$$
((\boldsymbol{w}, \boldsymbol{\beta}),(\boldsymbol{u}, \boldsymbol{\alpha}))+(\boldsymbol{A}(\boldsymbol{w}, \boldsymbol{\beta}),(\boldsymbol{u}, \boldsymbol{\alpha}))=((\boldsymbol{v}, \boldsymbol{\gamma}),(\boldsymbol{u}, \boldsymbol{\alpha})), \quad \forall(\boldsymbol{u}, \boldsymbol{\alpha}) \in \boldsymbol{H}
$$

If $(\boldsymbol{u}, \boldsymbol{\alpha}) \in \boldsymbol{V}$, then, (62) can be transformed in

$$
((\boldsymbol{w}, \boldsymbol{\beta}),(\boldsymbol{u}, \boldsymbol{\alpha}))+2 v \int_{\Omega_{F}} \boldsymbol{\varepsilon}(\boldsymbol{w}): \boldsymbol{\varepsilon}(\boldsymbol{u}) d \boldsymbol{y}=((\boldsymbol{v}, \boldsymbol{\gamma}),(\boldsymbol{u}, \boldsymbol{\alpha})), \quad \forall(\boldsymbol{u}, \boldsymbol{\alpha}) \in \boldsymbol{V}
$$

Using the Korn inequality (see [14, Theorem 2.4-2, p. 51]) and the Riesz theorem we deduce the existence and uniqueness of $(\boldsymbol{w}, \boldsymbol{\beta}) \in \boldsymbol{V}$ satisfying (63).

Taking $\boldsymbol{\alpha}=\mathbf{0}$ in (63), it is not difficult to see that $\boldsymbol{w}$ is solution of a Stokes-type system

$$
\left\{\begin{array}{lll}
\boldsymbol{w}-v \boldsymbol{\Delta} \boldsymbol{w}+\nabla \pi=\boldsymbol{F} & \text { in } & \Omega_{F} \\
\operatorname{div}(\boldsymbol{w})=0 & \text { in } & \Omega_{F} \\
\boldsymbol{w}=\mathbf{0} & \text { on } & \partial \Omega \\
\boldsymbol{w}=\sum_{i=1}^{N_{0}} \beta_{i} \boldsymbol{\xi}_{i} & \text { on } & \partial \Omega_{S} .
\end{array}\right.
$$

Since $\left\{\boldsymbol{\xi}_{i}\right\}_{i=1}^{N_{0}} \subset \boldsymbol{H}^{3}\left(\Omega_{S}\right)$ and $\partial \Omega \in \boldsymbol{C}^{2}$, we obtain $\boldsymbol{w} \in \boldsymbol{H}^{2}\left(\Omega_{F}\right)$. Therefore Id $+\boldsymbol{A}$ is onto.

This concludes that the operator $\boldsymbol{A}$ is self-adjoint (see, for instance [17, Proposition 3.2.4, p. 74]). Furthermore, $\boldsymbol{A}$ is non-negative, since from (61) we have

$$
(\boldsymbol{A}(\boldsymbol{w}, \boldsymbol{\beta}),(\boldsymbol{w}, \boldsymbol{\beta}))=2 v \int_{\Omega_{F}}\|\boldsymbol{\varepsilon}(\boldsymbol{w})\|^{2} d \boldsymbol{y} \geqslant 0 .
$$

Hence, thanks to Lumer-Phillips theorem, $\boldsymbol{A}$ is the generator of a contraction semigroup on $\boldsymbol{H}$ (see Proposition 3.3.5 and Proposition 3.8.4 in [17]). 
Using Proposition 4.1 and classical results on parabolic equations (see, for instance, [16]) we deduce the following result on (58)-(59):

PRoposition 4.2 For any $\left(\boldsymbol{w}^{0}, \boldsymbol{\beta}^{0}\right) \in \boldsymbol{V}$, for any $(\boldsymbol{F}, \boldsymbol{f}) \in \boldsymbol{L}^{2}\left(0, T ; \boldsymbol{L}^{2}\left(\Omega_{F}\right) \times \mathbb{R}^{N_{0}}\right)$ there exists a unique solution of (58)-(60) such that

$$
\widetilde{\boldsymbol{w}} \in D, \nabla \widetilde{\pi} \in \boldsymbol{L}^{2}\left(0, T ; \boldsymbol{L}^{2}\left(\Omega_{F}\right)\right), \widetilde{\boldsymbol{\beta}} \in\left[H^{1}(0, T)\right]^{N_{0}} .
$$

Moreover, we have the following estimate

$$
\begin{aligned}
\|\widetilde{\boldsymbol{w}}\|_{\Phi}+\|\nabla \widetilde{\pi}\|_{\boldsymbol{L}^{2}\left(0, T ; \boldsymbol{L}^{2}\left(\Omega_{F}\right)\right)}+\|\widetilde{\boldsymbol{\beta}}\|_{\left[\mathcal{H}{ }^{1}(0, T)\right]^{N_{0}}} \\
\quad \leqslant C\left(\|\boldsymbol{F}\|_{\boldsymbol{L}^{2}\left(0, T ; \boldsymbol{L}^{2}\left(\Omega_{F}\right)\right)}+\|\boldsymbol{f}\|_{\left[L^{2}(0, T)\right]^{N_{0}}}+\left\|\boldsymbol{w}^{0}\right\|_{\boldsymbol{H}^{1}\left(\Omega_{F}\right)}+\left\|\boldsymbol{\beta}^{0}\right\|_{\mathbb{R}^{N_{0}}}\right)
\end{aligned}
$$

where $C$ is a constant depending of $T$ in a non-decreasing way.

\section{Proof of the main result}

This section is devoted to the proof of Theorem 1.1. More precisely, we prove this theorem by using two technical results (Theorem 5.1 and Theorem 5.2) which will be proved in the next sections.

First let us fix $(\boldsymbol{w}, \pi, \boldsymbol{\beta})$ with

$$
\|\boldsymbol{w}\|_{Ð}+\|\nabla \pi\|_{\boldsymbol{L}^{2}\left(0, T ; \boldsymbol{L}^{2}\left(\Omega_{F}\right)\right)}+\|\boldsymbol{\beta}\|_{\left[\mathcal{H}^{1}(0, T)\right]^{N_{0}}} \leqslant R,
$$

where $R>0$ is a fixed positive constant.

Then we define $\alpha_{i}\left(i=1, \ldots, N_{0}\right)$ by

$$
\alpha_{i}(t)=\int_{0}^{t} \beta_{i}(s) d s
$$

In particular, if we take

$$
T=\frac{r_{1}}{2 R}
$$

we have for all $t,\left(\alpha_{1}(t), \ldots, \alpha_{N_{0}}(t)\right) \in B\left(\mathbf{0}, r_{1}\right)$, with $r_{1}$ such that we can apply Lemmata 2.1 and 2.4 and so that (17) holds true. By using these functions, we can define $\boldsymbol{\xi}, \widehat{\boldsymbol{\xi}_{i}}, \boldsymbol{X}, \widehat{\boldsymbol{\eta}_{i}},\left(\boldsymbol{W}_{i}, \pi_{i}\right)$ by the formulas (5), (19), (23), (44), and (45). Finally we can define $\boldsymbol{F}$ and $f_{i}$ by (57) and (52). Then we have the two following results.

THEOREM 5.1 Assume that $(\boldsymbol{w}, \pi, \boldsymbol{\beta})$ satisfies (65) and assume (66). Then there exists a positive constant $C(R)$ such that

$$
\|\boldsymbol{F}\|_{\boldsymbol{L}^{2}\left(0, T ; \boldsymbol{L}^{2}\left(\Omega_{F}\right)\right)}+\|\boldsymbol{f}\|_{\left[L^{2}(0, T)\right]^{N_{0}}} \leqslant C(R) T^{1 / 4} .
$$

Theorem 5.2 Assume that $\left(\boldsymbol{w}^{(1)}, \pi^{(1)}, \boldsymbol{\beta}^{(1)}\right),\left(\boldsymbol{w}^{(2)}, \pi^{(2)}, \boldsymbol{\beta}^{(2)}\right)$ satisfies (65) and assume (66). For $k=1,2$, we can construct $\boldsymbol{F}^{(k)}$ and $\boldsymbol{f}^{(k)}$ as above from (57) and (52) with $\left(\boldsymbol{w}^{(k)}, \pi^{(k)}, \boldsymbol{\beta}^{(k)}\right)$ 
instead of $(\boldsymbol{w}, \pi, \boldsymbol{\beta})$. Then there exists a positive constant $C(R)$ such that

$$
\begin{array}{r}
\left\|\boldsymbol{F}^{(1)}-\boldsymbol{F}^{(2)}\right\|_{\boldsymbol{L}^{2}\left(0, T ; \boldsymbol{L}^{2}\left(\Omega_{F}\right)\right)}+\left\|\boldsymbol{f}^{(1)}-\boldsymbol{f}^{(2)}\right\|_{\left[L^{2}(0, T)\right]^{N_{0}}} \\
\leqslant C(R) T^{1 / 4}\left(\left\|\boldsymbol{w}^{(1)}-\boldsymbol{w}^{(2)}\right\|_{\Phi}+\left\|\nabla\left(\pi^{(1)}-\pi^{(2)}\right)\right\|_{\boldsymbol{L}^{2}\left(0, T ; \boldsymbol{L}^{2}\left(\Omega_{F}\right)\right)}\right. \\
\left.+\left\|\boldsymbol{\beta}^{(1)}-\boldsymbol{\beta}^{(2)}\right\|_{\left[\mathcal{H}^{1}(0, T)\right]^{N_{0}}}\right) .
\end{array}
$$

These two results are technical and will be proved in the next two sections. Admitting these results, we are in position to prove the main result. The proof is based on the Banach fixed point theorem. More precisely, let $T>0, R>0$, and let us define

$$
\mathcal{K}=\Phi \times L^{2}\left(0, T ; H^{1}\left(\Omega_{F}\right)\right) \times\left[H^{1}(0, T)\right]^{N_{0}},
$$

endowed with the norm

$$
\|(\boldsymbol{w}, \pi, \boldsymbol{\beta})\| \mathcal{X}:=\|\boldsymbol{w}\|_{Ð}+\|\pi\|_{L^{2}\left(0, T ; H^{1}\left(\Omega_{F}\right)\right)}+\|\boldsymbol{\beta}\|_{\left[\mathcal{H}^{1}(0, T)\right]^{N_{0}}},
$$

and

$$
\mathrm{C}=\left\{(\boldsymbol{w}, \pi, \boldsymbol{\beta}) \in \mathcal{K}:\|(\boldsymbol{w}, \pi, \boldsymbol{\beta})\|_{\mathcal{K}} \leqslant R\right\} .
$$

Clearly, $\mathcal{C}$ is a closed subset of $\mathcal{K}$. Let us define the mapping

$$
\begin{aligned}
& z: \quad e \rightarrow K \\
& (\boldsymbol{w}, \pi, \boldsymbol{\beta}) \mapsto(\widetilde{\boldsymbol{w}}, \widetilde{\pi}, \widetilde{\boldsymbol{\beta}})
\end{aligned}
$$

where $(\widetilde{\boldsymbol{w}}, \widetilde{\pi}, \widetilde{\boldsymbol{\beta}})$ is the solution of (58)-(60), with $\boldsymbol{F}$ and $\boldsymbol{f}$ defined by (57) and (52) from (w, $\boldsymbol{\pi}, \boldsymbol{\beta})$ (as in the beginning of this section). The fact that $\mathcal{Z}$ maps $\mathcal{C}$ into $\mathcal{K}$ comes from Proposition 4.2 and Theorem 5.1. More precisely, applying Proposition 4.2, we obtain

$$
\|(\widetilde{\boldsymbol{w}}, \widetilde{\pi}, \widetilde{\boldsymbol{\beta}})\|_{\mathcal{X}} \leqslant C\left(\|\boldsymbol{F}\|_{\boldsymbol{L}^{2}\left(0, T ; \boldsymbol{L}^{2}\left(\Omega_{F}\right)\right)}+\|\boldsymbol{f}\|_{\left[L^{2}(0, T)\right]^{N_{0}}}+\left\|\boldsymbol{w}^{0}\right\|_{\boldsymbol{H}^{1}\left(\Omega_{F}\right)}+\left\|\boldsymbol{\beta}^{0}\right\|_{\mathbb{R}^{N_{0}}}\right) .
$$

Combining the above estimate with (67), we deduce

$$
\|\mathcal{Z}(\boldsymbol{w}, \pi, \boldsymbol{\beta})\|_{\mathcal{X}} \leqslant C(R) T^{1 / 4}+C\left(\left\|\boldsymbol{w}^{0}\right\|_{\boldsymbol{H}^{1}\left(\Omega_{F}\right)}+\left\|\boldsymbol{\beta}^{0}\right\|_{\mathbb{R}^{N_{0}}}\right) .
$$

With the constant $C$ and $C(R)$ of the above inequality, we take $R$ big enough so that

$$
\left\|\boldsymbol{w}^{0}\right\|_{\boldsymbol{H}^{1}\left(\Omega_{F}\right)}+\left\|\boldsymbol{\beta}^{0}\right\|_{\mathbb{R}^{N_{0}}} \leqslant \frac{R}{2 C}
$$

and $T$ small enough so that (in addition to (66))

$$
C(R) T^{1 / 4} \leqslant \frac{R}{2} .
$$

Gathering (71), (72) and (73), we deduce $Z(\mathcal{C}) \subset \mathcal{C}$. 
Lastly, we prove that $Z: \mathcal{C} \rightarrow \mathcal{C}$ is a contraction.

Let $\left(\boldsymbol{w}^{(1)}, \pi^{(1)}, \boldsymbol{\beta}^{(1)}\right),\left(\boldsymbol{w}^{(2)}, \pi^{(2)}, \boldsymbol{\beta}^{(2)}\right) \in \mathrm{C}$. Then, according to Proposition 4.2

$$
\begin{aligned}
\| Z\left(\boldsymbol{w}^{(1)}, \pi^{(1)}, \boldsymbol{\beta}^{(1)}\right)-Z & \left(\boldsymbol{w}^{(2)}, \pi^{(2)}, \boldsymbol{\beta}^{(2)}\right) \|_{\mathcal{X}} \\
& =\left\|\left(\widetilde{\boldsymbol{w}}^{(1)}, \widetilde{\pi}^{(1)}, \widetilde{\boldsymbol{\beta}}^{(1)}\right)-\left(\widetilde{\boldsymbol{w}}^{(2)}, \widetilde{\pi}^{(2)}, \widetilde{\boldsymbol{\beta}}^{(2)}\right)\right\|_{\mathcal{K}} \\
& \leqslant\left\|\boldsymbol{F}^{(1)}-\boldsymbol{F}^{(2)}\right\|_{\boldsymbol{L}^{2}\left(0, T ; \boldsymbol{L}^{2}\left(\Omega_{F}\right)\right)}+\left\|\boldsymbol{f}^{(1)}-\boldsymbol{f}^{(2)}\right\|_{\left[L^{2}(0, T)\right]^{N_{0}}}
\end{aligned}
$$

where, for $k=1,2, \boldsymbol{F}^{(k)}$ and $\boldsymbol{f}^{(k)}$ are defined by (57) and (52) with $\left(\boldsymbol{w}^{(k)}, \pi^{(k)}, \boldsymbol{\beta}^{(k)}\right)$. Thus, according to Theorem 5.2, we have

$$
\begin{aligned}
\left\|Z\left(w^{(1)}, \pi^{(1)}, \beta^{(1)}\right)-Z\left(w^{(2)}, \pi^{(2)}, \beta^{(2)}\right)\right\|_{\mathcal{X}} \\
\quad \leq C(R) T^{1 / 4}\left\|\left(w^{(1)}, \pi^{(1)}, \beta^{(1)}\right)-\left(w^{(2)}, \pi^{(2)}, \beta^{(2)}\right)\right\|_{\mathcal{X}} .
\end{aligned}
$$

Thus for $T$ small enough, $\mathcal{Z}$ is a contraction on $\mathcal{C}$ and we deduce the local in time existence of a solution of (48), (51) and thus of a solution of (7)-(8) by using Proposition 3.2. This completes the proof.

\section{Estimates on the coefficients}

This section is devoted to the proof of Theorem 5.1; more precisely, we estimate here $\boldsymbol{f}$ and $\boldsymbol{F}$ defined by (52) and (57). Throughout this section, we fix $(\boldsymbol{w}, \pi, \boldsymbol{\beta})$ with

$$
\|\boldsymbol{w}\|_{\triangleright}+\|\nabla \pi\|_{\boldsymbol{L}^{2}\left(0, T ; \boldsymbol{L}^{2}\left(\Omega_{F}\right)\right)}+\|\boldsymbol{\beta}\|_{\left[\mathcal{H}^{1}(0, T)\right]^{N_{0}}} \leqslant R,
$$

where $R$ is a fixed positive constant (see Section 5). We assume (66) and we define $\alpha_{i},(i=$ $\left.0, \ldots, N_{0}\right), \boldsymbol{\xi}, \widehat{\boldsymbol{\xi}_{i}}, \boldsymbol{X}, \widehat{\boldsymbol{\eta}}_{i},\left(\boldsymbol{W}_{i}, \pi_{i}\right)$ by the formulas (5), (19), (23), (44), and (45) as in the beginning of Section 5.

In the estimates below, the constants $C(R)$ at stake may depend on $R$, the geometry, $v, N_{0}$, $\left\{\xi_{i}\right\}_{i=0}^{N_{0}}$, and on $T$. If they depend on $T$, it is in a nondecreasing way. Similarly the constants $C$ at stake are independent on $R$ but may depend on the geometry, $\nu, N_{0},\left\{\boldsymbol{\xi}_{i}\right\}_{i=0}^{N_{0}}$, and on $T$. If they depend on $T$, it is in a nondecreasing way.

We recall that from the above assumptions and from Lemma 2.4, $\boldsymbol{X} \in \boldsymbol{H}^{2}\left(0, T ; \boldsymbol{H}^{3}(\Omega)\right)$, $\boldsymbol{Y} \in \boldsymbol{L}^{\infty}\left(0, T ; \boldsymbol{H}^{3}(\Omega)\right) \cap \boldsymbol{W}^{1, \infty}\left(0, T ; \boldsymbol{H}^{2}(\Omega)\right) \cap \boldsymbol{H}^{2}\left(0, T ; \boldsymbol{H}^{1}(\Omega)\right)$ and $(\nabla \boldsymbol{X})^{-1} \in \boldsymbol{H}^{2}(0, T:$ $\left.\boldsymbol{H}^{2}(\Omega)\right)$.

Lemma 6.1 With the above assumptions, $\operatorname{Cof}(\nabla \boldsymbol{X}) \in \boldsymbol{H}^{2}\left(0, T ; \boldsymbol{H}^{2}(\Omega)\right)$ and for all $1 \leqslant i \leqslant N_{0}$, $\widehat{\boldsymbol{\eta}}_{i} \in \boldsymbol{H}^{2}\left(0, T ; \boldsymbol{H}^{2}\left(\Omega_{S}\right)\right)$. Moreover, we have the following estimates

$$
\begin{gathered}
\|\boldsymbol{X}-\mathbf{i d}\|_{L^{\infty}\left(0, T ; \boldsymbol{H}^{3}(\Omega)\right)} \leqslant C(R) T . \\
\|\operatorname{Cof}(\nabla \boldsymbol{X})-\mathbf{I d}\|_{L_{\left(0, T ; H^{2}(\Omega)\right)}^{\infty}} \leqslant C(R) T^{1 / 2}, \quad\|\operatorname{Cof}(\nabla \boldsymbol{X})\|_{\boldsymbol{H}^{2}\left(0, T ; \boldsymbol{H}^{2}(\Omega)\right)} \leqslant C(R), \\
\left\|\frac{1}{\operatorname{det} \nabla \boldsymbol{X}}\right\|_{L^{\infty}\left(0, T ; H^{2}\left(\Omega_{S}\right)\right)} \leqslant C(R),\left\|\frac{1}{\operatorname{det} \nabla \boldsymbol{X}}-1\right\|_{L_{\left(0, T ; H^{2}\left(\Omega_{S}\right)\right)}} \leqslant C(R) T^{1 / 2},
\end{gathered}
$$




$$
\begin{gathered}
\left\|(\nabla \boldsymbol{X})^{-1}\right\|_{\boldsymbol{L}^{\infty}\left(0, T ; \boldsymbol{H}^{2}\left(\Omega_{S}\right)\right)} \leqslant C(R),\left\|(\nabla \boldsymbol{X})^{-1}-\mathbf{I d}\right\|_{\boldsymbol{L}^{\infty}\left(0, T ; \boldsymbol{H}^{2}\left(\Omega_{S}\right)\right)} \leqslant C(R) T^{1 / 2}, \\
\|\boldsymbol{X}\|_{\boldsymbol{H}^{2}\left(0, T ; \boldsymbol{H}^{3}(\Omega)\right)}+\|\boldsymbol{Y}\|_{\boldsymbol{L}^{\infty}\left(0, T ; \boldsymbol{H}^{3}(\Omega)\right)}+\left\|\frac{\partial \boldsymbol{Y}}{\partial t}\right\|_{\boldsymbol{L}_{\left(0, T ; \boldsymbol{H}^{2}(\Omega)\right)}} \leqslant C(R) . \\
+\left\|\frac{\partial^{2} \boldsymbol{Y}}{\partial t^{2}}\right\|_{\boldsymbol{L}^{2}\left(0, T ; \boldsymbol{H}^{1}(\Omega)\right)} \leqslant C \widehat{\boldsymbol{\eta}}_{i}-\boldsymbol{\xi}_{i} \|_{\boldsymbol{L}_{\left(0, T ; \boldsymbol{H}^{2}\left(\Omega_{S}\right)\right)} \leqslant C(R) T^{1 / 2},\left\|\widehat{\boldsymbol{\eta}}_{i}-\boldsymbol{\xi}_{i}\right\|_{\boldsymbol{W}^{1, \infty}\left(0, T ; \boldsymbol{H}^{2}\left(\Omega_{S}\right)\right)} \leqslant C(R) .}
\end{gathered}
$$

Proof. First, we remark that since the entries of $\operatorname{Cof}(\nabla \boldsymbol{X})$ are of the form

$$
\frac{\partial X_{i}}{\partial y_{j}} \frac{\partial X_{k}}{\partial y_{l}}-\frac{\partial X_{m}}{\partial y_{n}} \frac{\partial X_{p}}{\partial y_{q}}
$$

with $i, j, k, l, m, n, p, q \in\{1,2,3\}$, the regularity of $\operatorname{Cof}(\nabla \boldsymbol{X})$ is a consequence of the fact that $\boldsymbol{H}^{2}\left(0, T ; \boldsymbol{H}^{2}(\Omega)\right)$ is an algebra. On the other hand, by using the definitions (19) and (44),

$$
\widehat{\boldsymbol{\eta}}_{i}=(\operatorname{Cof} \nabla \boldsymbol{X})^{t}\left(\xi_{i}-\frac{\varsigma_{i}}{\varsigma_{0}} \xi_{0}\right)
$$

with (see (16))

$$
\varsigma_{i}=\int_{\partial \Omega_{S}}(\operatorname{Cof} \nabla \boldsymbol{X})^{t} \boldsymbol{\xi}_{i} \cdot \boldsymbol{n}_{\boldsymbol{y}} d \boldsymbol{\gamma}_{\boldsymbol{y}}
$$

These two last relations imply $\widehat{\boldsymbol{\eta}}_{i} \in \boldsymbol{H}^{2}\left(0, T ; \boldsymbol{H}^{2}\left(\Omega_{S}\right)\right)$.

By using the definition (23) of $\boldsymbol{X}$, we have

$$
\|\boldsymbol{X}-\mathbf{i d}\|_{\boldsymbol{L}^{\infty}\left(0, T ; \boldsymbol{H}^{3}(\Omega)\right)} \leqslant\|\varepsilon(\xi)\|_{\boldsymbol{L}_{\left(0, T ; \boldsymbol{H}^{3}(\Omega)\right)}} \leqslant C\|\boldsymbol{\alpha}\|_{\left[L^{\infty}(0, T)\right]^{N_{0}}} \leqslant C(R) T,
$$

which yields (75).

Similarly,

This implies

$$
\left\|\frac{\partial \boldsymbol{X}}{\partial t}\right\|_{\boldsymbol{H}^{1}\left(0, T ; \boldsymbol{H}^{3}(\Omega)\right)}=\left\|\varepsilon\left(\frac{\partial \xi}{\partial t}\right)\right\|_{\boldsymbol{H}^{1}\left(0, T ; \boldsymbol{H}^{3}(\Omega)\right)} \leqslant C(R) .
$$

$$
\|\boldsymbol{X}\|_{\boldsymbol{H}^{2}\left(0, T ; \boldsymbol{H}^{3}(\Omega)\right)} \leqslant C(R) .
$$

Combining (81) with (75) and (84), we deduce (76).

Let us prove (77). From (24), there exists a positive constant $C$ such that

$$
\|\operatorname{det}(\nabla \boldsymbol{Y})\|_{L^{\infty}\left(0, T ; L^{\infty}(\Omega)\right)} \leqslant C,
$$

and thus

$$
\left\|\frac{1}{\operatorname{det}(\nabla \boldsymbol{X})}\right\|_{L^{\infty}\left(0, T ; L^{\infty}(\Omega)\right)} \leqslant C .
$$

Combining the above estimate with (85) yields

$$
\left\|\frac{1}{\operatorname{det}(\nabla \boldsymbol{X})}\right\|_{L^{\infty}\left(0, T ; H^{2}(\Omega)\right)} \leqslant C\left(\|\nabla \boldsymbol{X}\|_{L_{\left(0, T ; \boldsymbol{H}^{2}(\Omega)\right)}^{2}}^{2}+1\right) \leqslant C(R) .
$$


On the other hand, from (75) and from the above estimate, we obtain

$$
\left\|\frac{1}{\operatorname{det} \nabla \boldsymbol{X}}-1\right\|_{L^{\infty}\left(0, T ; H^{2}(\Omega)\right)} \leqslant C(R)\|\nabla \boldsymbol{X}-\mathbf{I d}\|_{\boldsymbol{L}^{\infty}\left(0, T ; \boldsymbol{H}^{2}(\Omega)\right)} \leqslant C(R) T^{1 / 2} .
$$

This proves (77).

From (12), (76) and (77) we deduce (78).

Let us prove (79). To obtain the estimates on $\boldsymbol{Y}$, we follow the proof of Lemma 2.4. More precisely, from (24), there exists a positive constant $C$ such that

$$
\|\nabla Y\|_{L^{\infty}\left(0, T ; L^{\infty}(\Omega)\right)} \leqslant C .
$$

From (25), (77) and (78) we have

$$
\left\|\nabla^{2} \boldsymbol{Y}(\boldsymbol{X})\right\|_{\boldsymbol{L}^{\infty}\left(0, T ; \boldsymbol{L}^{6}(\Omega)\right)} \leqslant C(R) .
$$

Analogously, from (26), (77) and (78) we deduce

$$
\left\|\nabla^{3} \boldsymbol{Y}(\boldsymbol{X})\right\|_{\boldsymbol{L}^{\infty}\left(0, T ; \boldsymbol{L}^{2}(\Omega)\right)} \leqslant C(R) .
$$

Thus, we obtain

$$
\|\boldsymbol{Y}\|_{\boldsymbol{L}^{\infty}\left(0, T ; \boldsymbol{H}^{3}(\Omega)\right)} \leqslant C(R) .
$$

Then using (27) and the previous estimates on $\boldsymbol{X}$ and $\boldsymbol{Y}$, we obtain the estimates on $\frac{\partial \boldsymbol{Y}}{\partial t}$. We use the same kind of reasoning for the second derivative in time of $\boldsymbol{Y}$.

Lastly, we show (80), from (82) we deduce that

$$
\begin{aligned}
& \left\|\widehat{\boldsymbol{\eta}}_{i}-\boldsymbol{\xi}_{i}\right\|_{\boldsymbol{H}^{2}\left(\Omega_{S}\right)} \\
& \quad \leqslant\left\|(\operatorname{Cof} \nabla \boldsymbol{X})^{t}-\mathbf{I d}\right\|_{\boldsymbol{H}^{2}\left(\Omega_{S}\right)}\left\|\boldsymbol{\xi}_{i}\right\|_{\boldsymbol{H}^{2}\left(\Omega_{S}\right)}+\left\|(\operatorname{Cof} \nabla \boldsymbol{X})^{t}\right\|_{\boldsymbol{H}^{2}\left(\Omega_{S}\right)}\left\|\boldsymbol{\xi}_{0}\right\|_{\boldsymbol{H}^{2}\left(\Omega_{S}\right)}\left|\frac{\zeta_{i}(t)}{\zeta_{0}(t)}\right| .
\end{aligned}
$$

From (83) and (4), we have for all $i \in\left\{1, \ldots, N_{0}\right\}$,

$$
\varsigma_{i}=\int_{\partial \Omega_{S}}\left[(\mathbf{C o f} \nabla \boldsymbol{X})^{t}-\mathbf{I d}\right] \boldsymbol{\xi}_{i} \cdot \boldsymbol{n}_{\boldsymbol{y}} d \boldsymbol{\gamma}_{\boldsymbol{y}},
$$

and thus, by using (76), we have for all $i \in\left\{1, \ldots, N_{0}\right\}$,

$$
\left|\varsigma_{i}(t)\right| \leqslant C(R) T^{1 / 2} \text {. }
$$

Combining the above estimate with (18), (76), we conclude

$$
\left\|\widehat{\boldsymbol{\eta}}_{i}-\boldsymbol{\xi}_{i}\right\|_{\boldsymbol{L}^{\infty}\left(0, T ; \boldsymbol{H}^{2}\left(\Omega_{S}\right)\right)} \leqslant C(R) T^{1 / 2} .
$$

To obtain the estimates on the time derivative, we write

$$
\frac{\partial}{\partial t}\left(\widehat{\boldsymbol{\eta}}_{i}\right)=\frac{\partial}{\partial t}\left((\operatorname{Cof} \nabla \boldsymbol{X})^{t}\right) \boldsymbol{\xi}_{i}-\frac{\partial}{\partial t}\left((\operatorname{Cof} \nabla \boldsymbol{X})^{t}\right) \frac{\varsigma_{i}}{\varsigma_{0}} \boldsymbol{\xi}_{0}-(\operatorname{Cof} \nabla \boldsymbol{X})^{t} \frac{\partial}{\partial t}\left(\frac{\varsigma_{i}}{\varsigma_{0}}\right) \boldsymbol{\xi}_{0}
$$

and we use (76), (83) to deduce the result. 
LEMmA 6.2 Assume (74) and (66). Then for all $i \in\left\{1, \ldots, N_{0}\right\}$, the solution of (45) satisfies

$$
\left(\boldsymbol{W}_{i}, \pi_{i}\right) \in \boldsymbol{H}^{1}\left(0, T ; \boldsymbol{H}^{2}\left(\Omega_{F}\right)\right) \times H^{1}\left(0, T ; H^{1}\left(\Omega_{F}\right)\right)
$$

and there exists a constant $C(R)>0$ such that

$$
\left\|\boldsymbol{W}_{i}\right\|_{\mathcal{H}^{1}\left(0, T ; \boldsymbol{H}^{2}\left(\Omega_{F}\right)\right)}+\left\|\pi_{i}\right\|_{\mathcal{H}^{1}\left(0, T ; H^{1}\left(\Omega_{F}\right)\right)} \leqslant C(R) T^{1 / 2} .
$$

Proof. Using classical results (see [2]), we deduce that

$$
\left\|\boldsymbol{W}_{i}\right\|_{\boldsymbol{H}^{2}\left(\Omega_{F}\right)}+\left\|\pi_{i}\right\|_{H^{1}\left(\Omega_{F}\right) / \mathbb{R}} \leqslant C\left\|\widehat{\boldsymbol{\eta}}_{i}-\boldsymbol{\xi}_{i}\right\|_{\boldsymbol{H}^{3 / 2}\left(\partial \Omega_{S}\right)} \leqslant C\left\|\widehat{\boldsymbol{\eta}}_{i}-\boldsymbol{\xi}_{i}\right\|_{\boldsymbol{H}^{2}\left(\Omega_{S}\right)} .
$$

According to (80), this yields

$$
\left\|\boldsymbol{W}_{i}\right\|_{\boldsymbol{L}_{\left(0, T ; \boldsymbol{H}^{2}\left(\Omega_{F}\right)\right)}}+\left\|\pi_{i}\right\|_{L^{\infty}\left(0, T ; H^{1}\left(\Omega_{F}\right) / \mathbb{R}\right)} \leqslant C(R) T^{1 / 2} .
$$

Differentiating (45) with respect to time

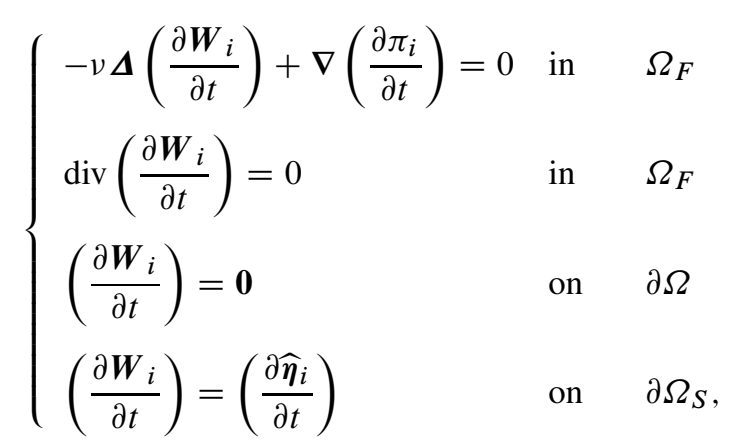

Using again the result of [2], we deduce

$$
\left\|\frac{\partial \boldsymbol{W}_{i}}{\partial t}\right\|_{\boldsymbol{H}^{2}\left(\Omega_{F}\right)}+\left\|\frac{\partial \pi_{i}}{\partial t}\right\|_{H^{1}\left(\Omega_{F}\right) / \mathbb{R}} \leqslant C\left\|\frac{\partial \widehat{\boldsymbol{\eta}}_{i}}{\partial t}\right\|_{\boldsymbol{H}^{3 / 2}\left(\partial \Omega_{S}\right)} \leqslant C\left\|\frac{\partial \widehat{\boldsymbol{\eta}}_{i}}{\partial t}\right\|_{\boldsymbol{H}^{2}\left(\Omega_{S}\right)} .
$$

Thus, thanks to (80), we have

$$
\left\|\boldsymbol{W}_{i}\right\|_{\boldsymbol{W}^{1, \infty}\left(0, T ; \boldsymbol{H}^{2}\left(\Omega_{F}\right)\right)}+\left\|\pi_{i}\right\|_{W^{1, \infty}\left(0, T ; H^{1}\left(\Omega_{F}\right)\right)} \leqslant C(R) .
$$

This inequality implies (87).

Using Lemma 6.1 the following result can be proved. We skip the proof since it is similar to the proof of next lemma.

LEMMA 6.3 Let $\boldsymbol{v} \in \Phi$ and $E_{i j}[\boldsymbol{v}]$ defined by (34). Then there exists a positive constant $C(R)$ such that

$$
\left\|E_{i j}[\boldsymbol{v}]\right\|_{L^{2}\left(0, T ; H^{1}\left(\Omega_{F}\right)\right)} \leqslant C(R) T^{1 / 2}\|\boldsymbol{v}\|_{\Phi} .
$$

LEMMA 6.4 Suppose that $v \in D$ and $q \in L^{2}\left(0, T ; H^{1}\left(\Omega_{F}\right)\right)$. Then there exists a positive constant $C(R)$ such that

(i) $\|[\boldsymbol{M} \boldsymbol{v}]\|_{\boldsymbol{L}^{2}\left(0, T ; \boldsymbol{L}^{2}\left(\Omega_{F}\right)\right)} \leqslant C(R) T^{1 / 2}\|\boldsymbol{v}\|_{Ð}$, 
(ii) $\|v([\boldsymbol{L} \boldsymbol{v}]-\boldsymbol{\Delta} \boldsymbol{v})\|_{\boldsymbol{L}^{2}\left(0, T ; \boldsymbol{L}^{2}\left(\Omega_{F}\right)\right)} \leqslant C(R) T^{1 / 2}\|\boldsymbol{v}\|_{\triangleright}$,

(iii) $\|[\boldsymbol{N} \boldsymbol{v}]\|_{\boldsymbol{L}^{2}\left(0, T ; \boldsymbol{L}^{2}\left(\Omega_{F}\right)\right)} \leqslant C(R) T^{1 / 4}\|\boldsymbol{v}\|_{\nexists}^{2}$,

(iv) $\|[\boldsymbol{G} q]-\nabla q\|_{L^{2}\left(0, T ; L^{2}\left(\Omega_{F}\right)\right)} \leqslant C(R) T^{1 / 2}\|q\|_{L^{2}\left(0, T ; H^{1}\left(\Omega_{F}\right)\right)}$,

(v) $\left\|\left[\boldsymbol{G}_{0}(\boldsymbol{v}, q)\right]\right\|_{L^{2}\left(0, T ; \boldsymbol{H}^{1}\left(\Omega_{F}\right)\right)} \leqslant C(R) T^{1 / 2}\left[1+\|\boldsymbol{v}\|_{\triangleright}+\|q\|_{L^{2}\left(0, T ; H^{1}\left(\Omega_{F}\right)\right)}\right]$.

Proof. By using the definition (39) and by performing some calculations, we have

$$
\begin{aligned}
{[\boldsymbol{M} v]_{i}=-\operatorname{tr}\left(( \boldsymbol { \nabla } \boldsymbol { X } ) ^ { - 1 } \left(\frac{\partial \boldsymbol{\nabla} \boldsymbol{X}}{\partial t}+\right.\right.} & \left.\left.\left(\nabla^{2} \boldsymbol{X}\right) \frac{\partial \boldsymbol{Y}}{\partial t}(\boldsymbol{X})\right)\right) \frac{\partial X_{i}}{\partial y_{k}} v_{k}+\sum_{k=1}^{3} \frac{\partial^{2} X_{i}}{\partial t \partial y_{k}} v_{k} \\
+ & \sum_{k, l=1}^{3} \frac{\partial^{2} X_{i}}{\partial y_{l} \partial y_{k}} \frac{\partial Y_{l}}{\partial t}(\boldsymbol{X}) v_{k} \\
& +\sum_{k, l=1}^{3} \frac{\partial X_{i}}{\partial y_{k}} \frac{\partial v_{k}}{\partial y_{l}} \frac{\partial Y_{l}}{\partial t}(\boldsymbol{X})+\sum_{k=1}^{3}\left(\frac{\partial X_{i}}{\partial y_{k}}-\delta_{i, k}\right) \frac{\partial v_{k}}{\partial t}
\end{aligned}
$$

where $\operatorname{tr}(\boldsymbol{A})$ denotes the trace of the matrix $\boldsymbol{A}$.

We estimate the first term in the above expression of $[\boldsymbol{M} v]_{i}$ :

$$
\begin{aligned}
\| \operatorname{tr}\left((\nabla \boldsymbol{X})^{-1}\right. & \left.\left(\frac{\partial \boldsymbol{\nabla} \boldsymbol{X}}{\partial t}+\left(\nabla^{2} \boldsymbol{X}\right) \frac{\partial \boldsymbol{Y}}{\partial t}(\boldsymbol{X})\right)\right) \frac{\partial X_{i}}{\partial y_{k}} v_{k} \|_{L^{2}\left(0, T ; L^{2}\left(\Omega_{F}\right)\right)} \\
& \leqslant T^{1 / 2}\left\|(\boldsymbol{\nabla} \boldsymbol{X})^{-1}\left(\frac{\partial \boldsymbol{\nabla} \boldsymbol{X}}{\partial t}+\left(\nabla^{2} \boldsymbol{X}\right) \frac{\partial \boldsymbol{Y}}{\partial t}(\boldsymbol{X})\right)\right\|_{\boldsymbol{L}^{\infty}\left(0, T ; \boldsymbol{L}^{\infty}\left(\Omega_{F}\right)\right)} \\
& \left\|\frac{\partial X_{i}}{\partial y_{k}}\right\|_{L^{\infty}\left(0, T ; L^{\infty}\left(\Omega_{F}\right)\right)}\left\|v_{k}\right\|_{L^{\infty}\left(0, T ; H^{1}\left(\Omega_{F}\right)\right)} .
\end{aligned}
$$

The above inequality combined with Lemma 6.1 yields

$$
\left\|\operatorname{tr}\left((\nabla \boldsymbol{X})^{-1}\left(\frac{\partial \boldsymbol{\nabla} \boldsymbol{X}}{\partial t}+\left(\nabla^{2} \boldsymbol{X}\right) \frac{\partial \boldsymbol{Y}}{\partial t}(\boldsymbol{X})\right)\right) \frac{\partial X_{i}}{\partial y_{k}} v_{k}\right\|_{L^{2}\left(0, T ; L^{2}\left(\Omega_{F}\right)\right)} \leqslant C(R) T^{1 / 2}\|\boldsymbol{v}\|_{\triangleright} .
$$

The three next terms in (92) are estimated in a similar way. For the last term, we write

$$
\begin{aligned}
\left\|\left(\frac{\partial X_{i}}{\partial y_{k}}-\delta_{i, k}\right) \frac{\partial v_{k}}{\partial t}\right\|_{L^{2}\left(0, T ; L^{2}\left(\Omega_{F}\right)\right)} & \leqslant\|\nabla \boldsymbol{X}-\mathbf{I d}\|_{\boldsymbol{L}_{\left(0, T ; \boldsymbol{L}^{\infty}\left(\Omega_{F}\right)\right)}\left\|\frac{\partial v_{k}}{\partial t}\right\|_{L^{2}\left(0, T ; L^{2}\left(\Omega_{F}\right)\right)}} \\
& \leqslant C(R) T\|\boldsymbol{v}\|_{\Phi},
\end{aligned}
$$

where the last inequality is obtained thanks to Lemma 6.1. All these estimates imply (i).

Now, we estimate the terms appearing in the expression (40) of $[\boldsymbol{L} \boldsymbol{v}]$. First,

$$
\begin{aligned}
\| \frac{\partial^{2} v_{i}}{\partial y_{j} \partial y_{l}}\left((\nabla \boldsymbol{X})_{l, j}^{-1}-\delta_{l, j}\right) & \|_{L^{2}\left(0, T ; L^{2}\left(\Omega_{F}\right)\right)} \\
& \leqslant\left\|(\nabla \boldsymbol{X})^{-1}-\mathbf{I d}\right\|_{\boldsymbol{L}^{\infty}\left(0, T ; \boldsymbol{L}^{\infty}\left(\Omega_{F}\right)\right)}\left\|v_{i}\right\|_{L^{2}\left(0, T ; H^{2}\left(\Omega_{F}\right)\right)} \\
& \leqslant C(R) T^{1 / 2}\|\boldsymbol{v}\|_{\Phi} .
\end{aligned}
$$


For the second term of $[\boldsymbol{L} \boldsymbol{v}]$, a short calculation shows that

$$
(\operatorname{det} \nabla \boldsymbol{X}) \frac{\partial}{\partial x_{j}}(\operatorname{det} \nabla \boldsymbol{Y})(\boldsymbol{X}) \frac{\partial v_{i}}{\partial y_{j}}=-\operatorname{tr}\left((\nabla \boldsymbol{X})^{-1}\left(\nabla^{2} \boldsymbol{X}\right)(\nabla \boldsymbol{X})^{-1}\right) \frac{\partial v_{i}}{\partial y_{j}},
$$

and then we estimate this term as the first term of $[\boldsymbol{M} v]$. Using Lemmata 6.1 and 6.3 we obtain the following estimate for the last term of $[\boldsymbol{L} \boldsymbol{v}]$ :

$$
\left\|(\operatorname{det} \nabla \boldsymbol{X}) \frac{\partial}{\partial x_{j}}\left(E_{i j}[\boldsymbol{v}]\right)(\boldsymbol{X})\right\|_{L^{2}\left(0, T ; L^{2}\left(\Omega_{F}\right)\right)} \leqslant C(R) T^{1 / 2}\|\boldsymbol{v}\|_{\triangleright} .
$$

These estimates allow to obtain (ii).

Next, we estimate each term in the expression (41) of $[\boldsymbol{N} \boldsymbol{v}]$. Since $\frac{\partial v_{i}}{\partial y_{j}} \in L^{\infty}\left(0, T ; L^{2}\left(\Omega_{F}\right)\right) \cap$ $L^{2}\left(0, T ; H^{1}\left(\Omega_{F}\right)\right)$ and since

$$
\left\|\frac{\partial v_{i}}{\partial y_{j}}\right\|_{H^{1 / 2}\left(\Omega_{F}\right)} \leqslant C\left\|\frac{\partial v_{i}}{\partial y_{j}}\right\|_{H^{1}\left(\Omega_{F}\right)}^{1 / 2}\left\|\frac{\partial v_{i}}{\partial y_{j}}\right\|_{L^{2}\left(\Omega_{F}\right)}^{1 / 2},
$$

we deduce that $\frac{\partial v_{i}}{\partial y_{j}} \in L^{4}\left(0, T ; H^{1 / 2}(\Omega)\right)$ and thus, using the Sobolev embedding theorem, that $\frac{\partial v_{i}}{\partial y_{j}} \in L^{4}\left(0, T ; L^{3}\left(\Omega_{F}\right)\right)$ with

$$
\left\|\frac{\partial v_{i}}{\partial y_{j}}\right\|_{L^{4}\left(0, T ; L^{3}\left(\Omega_{F}\right)\right)} \leqslant C\|\boldsymbol{v}\|_{\triangleright} .
$$

Hence, using the Hölder inequality and Lemma 6.1, we deduce

$$
\begin{aligned}
\left\|\frac{1}{\operatorname{det} \nabla \boldsymbol{X}} \sum_{j=1}^{3} \frac{\partial v_{i}}{\partial y_{j}} \sum_{m=1}^{3} \frac{\partial X_{j}}{\partial y_{m}} v_{m}\right\| & L^{2}\left(0, T ; L^{2}\left(\Omega_{F}\right)\right) \\
& \leqslant T^{1 / 4}\left\|\frac{1}{\operatorname{det} \nabla \boldsymbol{X}} \sum_{j=1}^{3} \frac{\partial v_{i}}{\partial y_{j}} \sum_{m=1}^{3} \frac{\partial X_{j}}{\partial y_{m}} v_{m}\right\|_{L^{4}\left(0, T ; L^{2}\left(\Omega_{F}\right)\right)}\left\|v_{m}\right\|_{L^{\infty}\left(0, T ; L^{6}\left(\Omega_{F}\right)\right)} \\
& \leqslant \sum_{j, m=1}^{3} T^{1 / 4}\left\|\frac{\partial v_{i}}{\partial y_{j}}\right\|_{L^{4}\left(0, T ; L^{3}\left(\Omega_{F}\right)\right)} \\
& \leqslant C(R) T^{1 / 4}\|\boldsymbol{v}\|_{\searrow}^{2} .
\end{aligned}
$$

Applying Lemmata 6.1 and 6.3, it can be shown that

$$
\left\|\sum_{j=1}^{3} E_{i j}[\boldsymbol{v}](\boldsymbol{X}) \sum_{m=1}^{3} \frac{\partial X_{j}}{\partial y_{m}} v_{m}\right\|_{L^{2}\left(0, T ; L^{2}\left(\Omega_{F}\right)\right)} \leqslant C(R) T^{1 / 2}\|\boldsymbol{v}\|_{\Phi}^{2} .
$$

Hence, we have proved (iii). 
Now, we estimate $\|[\boldsymbol{G} q]-\nabla q\|_{\boldsymbol{L}^{2}\left(0, T ; \boldsymbol{L}^{2}\left(\Omega_{F}\right)\right)}$ (see (42)). Some calculations give us the following inequalities

$$
\begin{aligned}
\left\|(\operatorname{det} \nabla \boldsymbol{X}) \frac{\partial}{\partial x_{i}}(\operatorname{det} \nabla \boldsymbol{Y})(\boldsymbol{X}) q\right\| & L^{2}\left(0, T ; L^{2}\left(\Omega_{F}\right)\right) \\
& =\left\|\operatorname{tr}\left((\boldsymbol{\nabla} \boldsymbol{X})^{-1}\left(\nabla^{2} \boldsymbol{X}\right)(\nabla \boldsymbol{X})^{-1}\right) q\right\|_{L^{2}\left(0, T ; L^{2}\left(\Omega_{F}\right)\right)} \\
& \leqslant C(R) T^{1 / 2}\|q\|_{L^{2}\left(0, T ; H^{1}\left(\Omega_{F}\right)\right)} .
\end{aligned}
$$

Finally, using Lemma 6.1 again

$$
\begin{aligned}
\left\|\sum_{l=1}^{3} \frac{\partial q}{\partial y_{l}}\left((\nabla \boldsymbol{X})_{l, i}^{-1}-\delta_{l, i}\right)\right\| & L^{2}\left(0, T ; L^{2}\left(\Omega_{F}\right)\right) \\
& \leqslant\left\|(\nabla \boldsymbol{X})^{-1}-\mathbf{I d}\right\|_{\boldsymbol{L}^{\infty}\left(0, T ; \boldsymbol{L}^{\infty}\left(\Omega_{F}\right)\right)}\|q\|_{L^{2}\left(0, T ; H^{1}\left(\Omega_{F}\right)\right)} \\
& \leqslant C(R) T^{1 / 2}\|q\|_{L^{2}\left(0, T ; H^{1}\left(\Omega_{F}\right)\right)} .
\end{aligned}
$$

Therefore,

$$
\|[\boldsymbol{G} q]-\nabla q\|_{\boldsymbol{L}^{2}\left(0, T ; \boldsymbol{L}^{2}\left(\Omega_{F}\right)\right)} \leqslant C(R) T^{1 / 2}\|q\|_{L^{2}\left(0, T ; H^{1}\left(\Omega_{F}\right)\right)} .
$$

Lastly, let us prove inequality (v) (see (50)). We estimate the first term, from Lemma 6.1 we deduce

$$
\begin{aligned}
\left\|\boldsymbol{\sigma}(\boldsymbol{v}, q)\left((\nabla \boldsymbol{X})^{-t}-\mathbf{I d}\right)\right\|_{\boldsymbol{L}^{2}\left(0, T ; \boldsymbol{H}^{1}\left(\Omega_{F}\right)\right)} & \\
& \leqslant\left\|(\nabla \boldsymbol{X})^{-t}-\mathbf{I d}\right\|_{\boldsymbol{L}^{\infty}\left(0, T ; \boldsymbol{H}^{2}\left(\Omega_{F}\right)\right)}\|\boldsymbol{\sigma}(\boldsymbol{v}, q)\|_{\boldsymbol{L}^{2}\left(0, T ; \boldsymbol{H}^{1}\left(\Omega_{F}\right)\right)} \\
& \leqslant C(R) T^{1 / 2}\left(\|\boldsymbol{v}\|_{\triangleright}+\|q\|_{L^{2}\left(0, T ; H^{1}\left(\Omega_{F}\right)\right)}\right) .
\end{aligned}
$$

For the second term, from Lemma 6.2 and (91), we have:

$$
\left\|\sigma\left(\boldsymbol{W}_{i}, \pi_{i}\right)\right\|_{L^{2}\left(0, T ; \boldsymbol{H}^{1}\left(\Omega_{F}\right)\right)} \leqslant C(R) T^{1 / 2}
$$

In a similar way to the estimates of the terms of $[\boldsymbol{L} \boldsymbol{v}]$ and thanks to Lemma 6.1 and (93), we deduce

$$
\left\|2 v\left(E[v]+(\boldsymbol{E}[\boldsymbol{v}])^{t}\right)(\boldsymbol{X}) \operatorname{Cof}(\nabla \boldsymbol{X})\right\|_{L^{2}\left(0, T ; \boldsymbol{H}^{1}\left(\Omega_{F}\right)\right)} \leqslant C(R) T^{1 / 2}\|\boldsymbol{v}\|_{\triangleright} .
$$

Applying Lemma 6.2, using (74) and similar calculations as in the term above, we obtain

$$
\left\|2 v \sum_{i=1}^{N_{0}} \beta_{i}\left(\boldsymbol{E}\left[\boldsymbol{W}_{i}\right]+\left(\boldsymbol{E}\left[\boldsymbol{W}_{i}\right]\right)^{t}\right)(\boldsymbol{X}) \operatorname{Cof}(\nabla \boldsymbol{X})\right\|_{\boldsymbol{L}^{2}\left(0, T ; \boldsymbol{H}^{1}\left(\Omega_{F}\right)\right)} \leqslant C(R) T^{1 / 2}
$$

This completes the proof of Lemma 6.4.

We are now in position to prove Theorem 5.1. 
Proof. We recall that

$$
\begin{aligned}
\boldsymbol{F}=-\sum_{i=1}^{N_{0}} \beta_{i}^{\prime} \boldsymbol{W}_{i}-\sum_{i=1}^{N_{0}} \beta_{i} \boldsymbol{W}_{i}^{\prime}-\sum_{i=1}^{N_{0}}\left[\boldsymbol{M}\left(\beta_{i} \boldsymbol{W}_{i}\right)\right] & \\
+v \sum_{i=1}^{N_{0}}\left[\boldsymbol{L}\left(\beta_{i} \boldsymbol{W}_{i}\right)\right]-\sum_{i=1}^{N_{0}}\left[\boldsymbol{G}\left(\beta_{i} \pi_{i}\right)\right]- & {\left[\boldsymbol{N}\left(\boldsymbol{w}+\sum_{i=1}^{N_{0}} \beta_{i} \boldsymbol{W}_{i}\right)\right] } \\
- & -[\boldsymbol{M} \boldsymbol{w}]+v[(\boldsymbol{L}-\boldsymbol{\Delta}) \boldsymbol{w}]+[(\nabla-\boldsymbol{G}) \pi] .
\end{aligned}
$$

First, we notice that

$$
\left\|\sum_{i=1}^{N_{0}} \beta_{i}^{\prime} \boldsymbol{W}_{i}\right\|_{\boldsymbol{L}^{2}\left(0, T ; \boldsymbol{L}^{2}\left(\Omega_{F}\right)\right)} \leqslant\|\boldsymbol{\beta}\|_{\left[H^{1}(0, T)\right]^{N_{0}}} \sum_{i=1}^{N_{0}}\left\|\boldsymbol{W}_{i}\right\|_{\boldsymbol{L}^{\infty}\left(0, T ; \boldsymbol{L}^{2}\left(\Omega_{F}\right)\right)} \leqslant C(R) T^{1 / 2},
$$

according to (74) and (87). The second term of $\boldsymbol{F}$ is bounded in the same way:

$$
\left\|\sum_{i=1}^{N_{0}} \beta_{i} \boldsymbol{W}_{i}^{\prime}\right\|_{\boldsymbol{L}^{2}\left(0, T ; \boldsymbol{L}^{2}\left(\Omega_{F}\right)\right)} \leqslant\|\boldsymbol{\beta}\|_{\left[\boldsymbol{L}^{\infty}(0, T)\right]^{N_{0}}} \sum_{i=1}^{N_{0}}\left\|\boldsymbol{W}_{i}\right\|_{\boldsymbol{H}^{1}\left(0, T ; \boldsymbol{L}^{2}\left(\Omega_{F}\right)\right)} \leqslant C(R) T^{1 / 2} .
$$

Thanks to Lemma 6.4 (i), (74) and (87), we have

$$
\begin{aligned}
\left\|\sum_{i=1}^{N_{0}}\left[\boldsymbol{M}\left(\beta_{i} \boldsymbol{W}_{i}\right)\right]\right\|_{\boldsymbol{L}^{2}\left(0, T ; \boldsymbol{L}^{2}\left(\Omega_{F}\right)\right)} & \leqslant C(R) T^{1 / 2}\left\|\sum_{i=1}^{N_{0}} \beta_{i} \boldsymbol{W}_{i}\right\|_{Ð} \\
& \leqslant C(R) T^{1 / 2}\|\boldsymbol{\beta}\|_{[\mathcal{H}(0, T)]^{N_{0}}} \sum_{i=1}^{N_{0}}\left\|\boldsymbol{W}_{i}\right\|_{\boldsymbol{H}^{1}\left(0, T ; \boldsymbol{H}^{2}\left(\Omega_{F}\right)\right)} \\
& \leqslant C(R) T .
\end{aligned}
$$

According to Lemma 6.4 (ii) and (iv), (74) and (87), we have

$$
\begin{aligned}
& \left\|v \sum_{i=1}^{N_{0}}\left[\boldsymbol{L}\left(\beta_{i} \boldsymbol{W}_{i}\right)\right]-\sum_{i=1}^{N_{0}}\left[\boldsymbol{G}\left(\beta_{i} \pi_{i}\right)\right]\right\|_{\boldsymbol{L}^{2}\left(0, T ; \boldsymbol{L}^{2}\left(\Omega_{F}\right)\right)} \\
& \leqslant\left\|v \sum_{i=1}^{N_{0}}\left(\left[\boldsymbol{L}\left(\beta_{i} \boldsymbol{W}_{i}\right)\right]-\beta_{i} \boldsymbol{\Delta} \boldsymbol{W}_{i}\right)\right\|_{\boldsymbol{L}^{2}\left(0, T ; \boldsymbol{L}^{2}\left(\Omega_{F}\right)\right)} \\
& +\left\|\sum_{i=1}^{N_{0}}\left(\nabla\left(\beta_{i} \pi_{i}\right)-\left[\boldsymbol{G}\left(\beta_{i} \pi_{i}\right)\right]\right)\right\|_{\boldsymbol{L}^{2}\left(0, T ; \boldsymbol{L}^{2}\left(\Omega_{F}\right)\right)} \\
& \leqslant C(R) T^{1 / 2}\left\|\sum_{i=1}^{N_{0}} \beta_{i} \boldsymbol{W}_{i}\right\|_{\searrow}+C(R) T^{1 / 2}\left\|\sum_{i=1}^{N_{0}} \beta_{i} \pi_{i}\right\|_{\left.L^{2}\left(0, T ; H^{1}\left(\Omega_{F}\right)\right)\right)} \leqslant C(R) T .
\end{aligned}
$$


Using Lemma 6.4 (iii), (74) and (87)

$$
\begin{aligned}
\left\|\left[\boldsymbol{N}\left(\boldsymbol{w}+\sum_{i=1}^{N_{0}} \beta_{i} \boldsymbol{W}_{i}\right)\right]\right\| & \boldsymbol{L}^{2}\left(0, T ; \boldsymbol{L}^{2}\left(\Omega_{F}\right)\right) \\
& \leqslant C(R) T^{1 / 4}\left\|\boldsymbol{w}+\sum_{i=1}^{N_{0}} \beta_{i} \boldsymbol{W}_{i}\right\|_{\Phi}^{2} \\
& \leqslant C(R) T^{1 / 4}\left(\|\boldsymbol{w}\|_{\Phi}^{2}+\|\boldsymbol{\beta}\|_{[\mathcal{H} 1(0, T)]^{N_{0}}}^{2} \sum_{i=1}^{N_{0}}\left\|\boldsymbol{W}_{i}\right\|_{\boldsymbol{H}^{1}\left(0, T ; \boldsymbol{H}^{2}\left(\Omega_{F}\right)\right)}^{2}\right) \\
& \leqslant C(R) T^{1 / 4} .
\end{aligned}
$$

Moreover, using again Lemma 6.4 (i), (ii) and (iv), we have

$$
\begin{aligned}
\|-[\boldsymbol{M} \boldsymbol{w}]+v[(\boldsymbol{L}-\boldsymbol{\Delta}) \boldsymbol{w}]+[(\boldsymbol{\nabla}-\boldsymbol{G}) \pi]\|_{\boldsymbol{L}^{2}\left(0, T ; \boldsymbol{L}^{2}\left(\Omega_{F}\right)\right)} & \leqslant C(R) T^{1 / 2}\left(\|\boldsymbol{w}\|_{\triangleright}+\|\pi\|_{L^{2}\left(0, T ; H^{1}\left(\Omega_{F}\right)\right)}\right) \\
& \leqslant C(R) T^{1 / 2} .
\end{aligned}
$$

All these inequalities imply that

$$
\|\boldsymbol{F}\|_{\boldsymbol{L}^{2}\left(0, T ; \boldsymbol{L}^{2}\left(\Omega_{F}\right)\right)} \leqslant C(R) T^{1 / 4} .
$$

On the other hand, $\boldsymbol{f}$ is defined by (52). First, note that by (19), Lemma 2.5 and (86) we have

$$
\left\|\widehat{\boldsymbol{\xi}}_{i}-\boldsymbol{\xi}_{i}\right\|_{\boldsymbol{L}_{\left(0, T ; \boldsymbol{H}^{3}\left(\Omega_{S}\right)\right)}} \leqslant C(R) T^{1 / 2} .
$$

Thereby, we deduce

$$
\begin{aligned}
& \left\|\sum_{j=1}^{N_{0}} \beta_{j}^{\prime} \int_{\Omega_{S}}\left(\widehat{\boldsymbol{\xi}}_{j}-\boldsymbol{\xi}_{j}\right) \cdot \boldsymbol{\xi}_{i} d \boldsymbol{y}\right\|_{L^{2}(0, T)} \leqslant C(R) T^{1 / 2}\left\|\boldsymbol{\beta}^{\prime}\right\|_{L^{2}(0, T)} \leqslant C(R) T^{1 / 2}, \\
& \left\|\sum_{j=1}^{N_{0}} \beta_{j}^{\prime} \int_{\Omega_{S}} \widehat{\boldsymbol{\xi}}_{j} \cdot\left(\widehat{\boldsymbol{\xi}}_{i}-\boldsymbol{\xi}_{i}\right) d \boldsymbol{y}\right\|_{L^{2}(0, T)} \leqslant C(R) T^{1 / 2}\left\|\beta^{\prime}\right\|_{L^{2}(0, T)} \leqslant C(R) T^{1 / 2},
\end{aligned}
$$

and

$$
\left\|\int_{\partial \Omega_{S}} \boldsymbol{\sigma}(\boldsymbol{w}, \pi) \boldsymbol{n}_{\boldsymbol{y}} \cdot\left(\widehat{\boldsymbol{\xi}}_{i}-\boldsymbol{\xi}_{i}\right) d \boldsymbol{\gamma}_{\boldsymbol{y}}\right\|_{L^{2}(0, T)} \leqslant C(R) T^{1 / 2}\|\boldsymbol{\sigma}(\boldsymbol{w}, \pi)\|_{\boldsymbol{L}^{2}\left(0, T ; \boldsymbol{H}^{1}\left(\Omega_{F}\right)\right)} \leqslant C(R) T^{1 / 2}
$$

For the second term, we have

$$
\left\|\sum_{j=1}^{N_{0}} \beta_{j} \int_{\Omega_{S}} \frac{\partial}{\partial t}\left(\widehat{\boldsymbol{\xi}}_{j}\right) \cdot \widehat{\boldsymbol{\xi}}_{i} d \boldsymbol{y}\right\|_{L^{2}(0, T)} \leqslant C(R)\|\boldsymbol{\beta}\|_{L^{2}(0, T)} \leqslant C(R) T^{1 / 2} .
$$


On the other hand, using (19) and (86)

$$
\begin{aligned}
2 \mu\left\|\int_{\Omega_{S}} \boldsymbol{\varepsilon}(\boldsymbol{\xi}): \boldsymbol{\varepsilon}\left(\widehat{\boldsymbol{\xi}}_{i}\right) d \boldsymbol{y}\right\|_{L^{2}(0, T)} & +\lambda\left\|\int_{\Omega_{S}} \operatorname{div}(\boldsymbol{\xi}) \operatorname{div}\left(\widehat{\boldsymbol{\xi}}_{i}\right) d \boldsymbol{y}\right\|_{L^{2}(0, T)} \\
& \leqslant C(R)\left(1+T^{1 / 2}\right)\|\boldsymbol{\alpha}\|_{L^{2}(0, T)} \\
& \leqslant C(R) T^{3 / 2}
\end{aligned}
$$

Finally, using Lemma 6.4 (v)

$$
\left\|\int_{\partial \Omega_{S}} \boldsymbol{G}_{0}[\boldsymbol{w}, \pi] \cdot \widehat{\boldsymbol{\xi}}_{i} d \boldsymbol{\gamma}_{\boldsymbol{y}}\right\|_{L^{2}(0, T)} \leqslant C(R) T^{1 / 2}
$$

and thus

$$
\|\boldsymbol{f}\|_{\left[L^{2}(0, T)\right]^{N_{0}}} \leqslant C(R) T^{1 / 2}
$$

The proof of Theorem 5.1 concludes by combining the above equation and (95).

\section{Estimates on the differences of the operators}

This section is devoted to the proof of Theorem 5.2; more precisely, we estimate the differences $\boldsymbol{F}^{(1)}-\boldsymbol{F}^{(2)}$ and $\boldsymbol{f}^{(1)}-\boldsymbol{f}^{(2)}$. Throughout this section, for $k \in\{1,2\}$ we fix $\left(\boldsymbol{w}^{(k)}, \pi^{(k)}, \boldsymbol{\beta}^{(k)}\right)$ with

$$
\left\|\boldsymbol{w}^{(k)}\right\|_{\Phi}+\left\|\nabla \pi^{(k)}\right\|_{L^{2}\left(0, T ; L^{2}\left(\Omega_{F}\right)\right)}+\left\|\boldsymbol{\beta}^{(k)}\right\|_{\left[\mathcal{H}^{1}(0, T)\right]^{N_{0}}} \leqslant R
$$

where $R$ is a fixed positive constant (see Section 5). Here $\boldsymbol{f}^{(k)}$ and $\boldsymbol{F}^{(k)}$ are defined by (52) and (57). More precisely, we assume (66) and by using these families of functions we can define $\alpha_{i}^{(k)}$, $\left(i=0, \ldots, N_{0}\right), \boldsymbol{\xi}^{(k)}, \widehat{\boldsymbol{\xi}}_{i}^{(k)}, \boldsymbol{X}^{(k)}, \widehat{\boldsymbol{\eta}}_{i}^{(k)},\left(\boldsymbol{W}_{i}^{(k)}, \pi_{i}^{(k)}\right)$ by the formulas (5), (19), (23), (44), and (45) as in the beginning of Section 5.

In the estimates below, the constants $C(R)$ at stake may depend on $R$, the geometry, $v, N_{0}$, $\left\{\boldsymbol{\xi}_{i}\right\}_{i=0}^{N_{0}}$, and on $T$. If they depend on $T$, it is in a nondecreasing way. Similarly the constants $C$ at stake are independent on $R$ but may depend on the geometry, $v, N_{0},\left\{\boldsymbol{\xi}_{i}\right\}_{i=0}^{N_{0}}$, and on $T$. If they depend on $T$, it is in a nondecreasing way.

We recall that from the above assumptions and from Lemma 2.4, for $n \in\{1,2\}, \boldsymbol{X}^{(k)} \in$ $\boldsymbol{H}^{2}\left(0, T ; \boldsymbol{H}^{3}(\Omega)\right), \boldsymbol{Y}^{(k)} \in \boldsymbol{L}^{\infty}\left(0, T ; \boldsymbol{H}^{3}(\Omega)\right) \cap \boldsymbol{W}^{1, \infty}\left(0, T ; \boldsymbol{H}^{2}(\Omega)\right) \cap \boldsymbol{H}^{2}\left(0, T ; \boldsymbol{H}^{1}(\Omega)\right)$ and $\left(\nabla \boldsymbol{X}^{(k)}\right)^{-1} \in \boldsymbol{H}^{2}\left(0, T: \boldsymbol{H}^{2}(\Omega)\right)$.

We first estimate the differences of the functions $\boldsymbol{X}$ and $\boldsymbol{Y}$ :

LEMmA 7.1 Assume $\left\{\beta_{i}^{(n)}\right\}_{i=1}^{N_{0}},(n \in\{1,2\})$ satisfy the above conditions. Then we have the 
following estimates

$$
\begin{aligned}
&\left\|\boldsymbol{X}^{(1)}-\boldsymbol{X}^{(2)}\right\|_{\boldsymbol{W}^{1, \infty}\left(0, T ; \boldsymbol{H}^{3}(\Omega)\right)}+\left\|\boldsymbol{Y}^{(1)}-\boldsymbol{Y}^{(2)}\right\|_{\boldsymbol{L}^{\infty}\left(0, T ; \boldsymbol{H}^{3}(\Omega)\right)} \\
&+\left\|\frac{\partial\left(\boldsymbol{Y}^{(1)}-\boldsymbol{Y}^{(2)}\right)}{\partial t}\right\|_{L_{\left(0, T ; \boldsymbol{H}^{2}(\Omega)\right)}^{\infty}}+\left\|\frac{\partial^{2}\left(\boldsymbol{Y}^{(1)}-\boldsymbol{Y}^{(2)}\right)}{\partial t^{2}}\right\| \\
& \leqslant C(R) T^{1 / 2}\left\|\boldsymbol{\beta}^{(1)}-\boldsymbol{\beta}^{(2)}\right\|_{\left[\boldsymbol{H}^{1}(0, T)\right]^{N_{0}}} .
\end{aligned}
$$

Proof. The proof is quite similar to the proof of Lemma 6.1 so we only precise the main changes. First using that the mapping $\phi$ in Lemma 2.1 is of class $C^{\infty}$, there exists a positive constant $C$ such that

$$
\left\|\alpha_{0}^{(1)}-\alpha_{0}^{(2)}\right\|_{H^{2}(0, T)} \leqslant C\left\|\boldsymbol{\alpha}^{(1)}-\boldsymbol{\alpha}^{(2)}\right\|_{\left[H^{2}(0, T)\right]^{N_{0}}} .
$$

Consequently, since $\boldsymbol{\alpha}^{(1)}(0)=\alpha^{(2)}(0)$

$$
\begin{aligned}
\left\|\varepsilon\left(\xi^{(1)}\right)-\varepsilon\left(\xi^{(2)}\right)\right\|_{\boldsymbol{L}^{\infty}\left(0, T ; \boldsymbol{L}^{\infty}(\Omega)\right)} & \leqslant C\left\|\boldsymbol{\alpha}^{(1)}-\boldsymbol{\alpha}^{(2)}\right\|_{\left[\boldsymbol{L}^{\infty}(0, T)\right]^{N_{0}}} \\
& \leqslant C T^{1 / 2}\left\|\boldsymbol{\beta}^{(1)}-\boldsymbol{\beta}^{(2)}\right\|_{\left[\mathcal{H}^{1}(0, T)\right]^{N_{0}}} .
\end{aligned}
$$

Therefore, by writing

$$
\boldsymbol{Y}^{(n)}(\boldsymbol{x})=-\varepsilon\left(\xi^{(n)}\right)\left(\boldsymbol{Y}^{(n)}(\boldsymbol{x})\right)+\boldsymbol{x}
$$

for $n=1,2$, we can estimate $\boldsymbol{Y}^{(1)}-\boldsymbol{Y}^{(2)}$ :

$$
\begin{aligned}
\left\|\boldsymbol{Y}^{(1)}-\boldsymbol{Y}^{(2)}\right\|_{\boldsymbol{L}^{\infty}\left(0, T ; \boldsymbol{L}^{\infty}(\Omega)\right)} & \leqslant \frac{\left\|\varepsilon\left(\xi^{(1)}\right)-\varepsilon\left(\xi^{(2)}\right)\right\|_{\boldsymbol{L}^{\infty}\left(0, T ; \boldsymbol{L}^{\infty}(\Omega)\right)}}{1-\left\|\varepsilon\left(\xi^{(2)}\right)\right\|_{\boldsymbol{L}^{\infty}\left(0, T ; \boldsymbol{W}^{1, \infty}(\Omega)\right)}} \\
& \leqslant \frac{C T\left\|\boldsymbol{\beta}^{(1)}-\boldsymbol{\beta}^{(2)}\right\|_{\left[\mathcal{H}^{1}(0, T)\right]^{N_{0}}}}{1-\left\|\varepsilon\left(\xi^{(2)}\right)\right\|_{L_{\left(0, T ; \boldsymbol{W}^{1, \infty}(\Omega)\right)}}}
\end{aligned}
$$

The other estimates on $\boldsymbol{Y}$ are proved by using the above estimate.

Finally, using the above lemma, we can prove the following results. Since their proofs are similar to the proofs of Lemma 6.1 and Lemma 6.4 (see also [16]), we skip them.

LEMMA 7.2 With the above assumptions, we have the following regularity results: there exist a positive constant $C(R)$ such that

$$
\left\|\operatorname{Cof}\left(\nabla X^{(1)}\right)-\operatorname{Cof}\left(\nabla X^{(2)}\right)\right\|_{W^{1, \infty}\left(0, T ; H^{2}(\Omega)\right)} \leqslant C(R) T^{1 / 2}\left\|\beta^{(1)}-\beta^{(2)}\right\|_{\left[\mathcal{H}^{1}(0, T)\right]^{N_{0}}},
$$


and for all $1 \leqslant i \leqslant N_{0}$ we have

$$
\left\|\widehat{\boldsymbol{\xi}}_{i}^{(1)}-\widehat{\boldsymbol{\xi}}_{i}^{(2)}\right\|_{\boldsymbol{W}^{1, \infty}\left(0, T ; \boldsymbol{H}^{3}\left(\Omega_{S}\right)\right)} \leqslant C(R) T^{1 / 2}\left\|\boldsymbol{\beta}^{(1)}-\boldsymbol{\beta}^{(2)}\right\|_{\left[\mathcal{H}^{1}(0, T)\right]^{N_{0}}},
$$

and

$$
\left\|\widehat{\boldsymbol{\eta}}_{i}^{(1)}-\widehat{\boldsymbol{\eta}}_{i}^{(2)}\right\|_{\boldsymbol{W}^{1, \infty}\left(0, T ; \boldsymbol{H}^{2}\left(\Omega_{S}\right)\right)} \leqslant C(R) T^{1 / 2}\left\|\boldsymbol{\beta}^{(1)}-\boldsymbol{\beta}^{(2)}\right\|_{\left[\mathcal{H}^{1}(0, T)\right]^{N_{0}}} .
$$

LEMma 7.3 Suppose that $v \in \Phi$ and $q \in L^{2}\left(0, T ; H^{1}\left(\Omega_{F}\right)\right)$. Then there exists a positive constant $C(R)$ such that

(i) $\left\|\left[\boldsymbol{M}^{(1)} \boldsymbol{v}\right]-\left[\boldsymbol{M}^{(2)} \boldsymbol{v}\right]\right\|_{\boldsymbol{L}^{2}\left(0, T ; \boldsymbol{L}^{2}\left(\Omega_{F}\right)\right)} \leqslant C(R) T^{1 / 2}\|\boldsymbol{v}\|_{\triangleright}\left\|\boldsymbol{\beta}^{(1)}-\boldsymbol{\beta}^{(2)}\right\|_{\left[\mathcal{H}^{1}(0, T)\right]^{N_{0}}}$,

(ii) $\left\|v\left(\left[\boldsymbol{L}^{(1)} \boldsymbol{v}\right]-\left[\boldsymbol{L}^{(2)} \boldsymbol{v}\right]\right)\right\|_{\boldsymbol{L}^{2}\left(0, T ; \boldsymbol{L}^{2}\left(\Omega_{F}\right)\right)} \leqslant C(R) T^{1 / 2}\|\boldsymbol{v}\|_{\triangleright}\left\|\boldsymbol{\beta}^{(1)}-\boldsymbol{\beta}^{(2)}\right\|_{\left[\mathcal{H}^{1}(0, T)\right]^{N_{0}}}$,

(iii) $\left\|\left[\boldsymbol{G}^{(1)} q\right]-\left[\boldsymbol{G}^{(2)} q\right]\right\|_{\boldsymbol{L}^{2}\left(0, T ; \boldsymbol{L}^{2}\left(\Omega_{F}\right)\right)}$

$$
\leqslant C(R) T^{1 / 2}\|q\|_{L^{2}\left(0, T ; H^{1}\left(\Omega_{F}\right)\right)}\left\|\boldsymbol{\beta}^{(1)}-\boldsymbol{\beta}^{(2)}\right\|_{\left[\mathcal{H}^{1}(0, T)\right]^{N_{0}}},
$$

(iv) $\left\|\boldsymbol{G}_{0}^{(1)}[\boldsymbol{v}, q]-\boldsymbol{G}_{0}^{(2)}[\boldsymbol{v}, q]\right\|_{\boldsymbol{L}^{2}\left(0, T ; \boldsymbol{H}^{1}\left(\Omega_{F}\right)\right)}$

$$
\leqslant C(R) T^{1 / 2}\left(\|\boldsymbol{v}\|_{\Phi}+\|q\|_{L^{2}\left(0, T ; H^{1}\left(\Omega_{F}\right)\right)}+\left\|\boldsymbol{\beta}^{(1)}-\boldsymbol{\beta}^{(2)}\right\|_{\left[\mathcal{H}^{1}(0, T)\right]^{N_{0}}}\right) .
$$

We also have the following consequence of Lemma 7.1:

Lemma 7.4 Suppose $\boldsymbol{v}^{(1)}, \boldsymbol{v}^{(2)} \in \Phi$. Then there exists a positive constant $C(R)$ such that

$$
\begin{aligned}
& \left\|\left[N^{(1)} v^{(1)}\right]-\left[N^{(2)} v^{(2)}\right]\right\|_{L^{2}\left(0, T ; L^{2}\left(\Omega_{F}\right)\right)} \\
& \leqslant C(R) T^{1 / 4}\left(\left\|\boldsymbol{v}^{(1)}\right\|_{Ð}+\left\|\boldsymbol{v}^{(2)}\right\|_{Ð}\right) \\
& \left(\left(\left\|\boldsymbol{v}^{(1)}\right\|_{\supset}+\left\|\boldsymbol{v}^{(2)}\right\|_{\supset}\right)\left\|\boldsymbol{\beta}^{(1)}-\boldsymbol{\beta}^{(2)}\right\|_{\left[\mathcal{H}^{1}(0, T)\right]^{N_{0}}}+\left\|\boldsymbol{v}^{(1)}-\boldsymbol{v}^{(2)}\right\|_{囚}\right) \text {. }
\end{aligned}
$$

Now we will prove Theorem 5.2.

Proof. Let $\left(\boldsymbol{w}^{(1)}, \pi^{(1)}, \boldsymbol{\beta}^{(1)}\right),\left(\boldsymbol{w}^{(2)}, \pi^{(2)}, \boldsymbol{\beta}^{(2)}\right)$ satisfying (96). For $k=1,2$, we define $\boldsymbol{F}^{(k)}$ and $f^{(k)}$ by (57) and (52) respectively. Then, we have:

$$
\begin{gathered}
\boldsymbol{F}^{(1)}-\boldsymbol{F}^{(2)}=-\sum_{i=1}^{N_{0}}\left(\beta_{i}^{(1)^{\prime}}-\beta_{i}^{(2)^{\prime}}\right) \boldsymbol{W}_{i}^{(1)}-\sum_{i=1}^{N_{0}} \beta_{i}^{(2)^{\prime}}\left(\boldsymbol{W}_{i}^{(1)}-\boldsymbol{W}_{i}^{(2)}\right) \\
-\sum_{i=1}^{N_{0}}\left(\beta_{i}^{(1)}-\beta_{i}^{(2)}\right) \boldsymbol{W}_{i}^{(1)^{\prime}}-\sum_{i=1}^{N_{0}} \beta_{i}^{(2)}\left(\boldsymbol{W}_{i}^{(1)^{\prime}}-\boldsymbol{W}_{i}^{(2)^{\prime}}\right) \\
-\sum_{i=1}^{N_{0}}\left(\left[\boldsymbol{M}^{(1)}\left(\beta_{i}^{(1)} \boldsymbol{W}_{i}^{(1)}\right)\right]-\left[\boldsymbol{M}^{(2)}\left(\beta_{i}^{(1)} \boldsymbol{W}_{i}^{(1)}\right)\right]\right) \\
-\sum_{i=1}^{N_{0}}\left[\boldsymbol{M}^{(2)}\left(\beta_{i}^{(1)} \boldsymbol{W}_{i}^{(1)}-\beta_{i}^{(2)} \boldsymbol{W}_{i}^{(2)}\right)\right]-v \sum_{i=1}^{N_{0}}\left(\left[\boldsymbol{L}^{(1)}\left(\beta_{i}^{(1)} \boldsymbol{W}_{i}^{(1)}\right)\right]\right.
\end{gathered}
$$




$$
\begin{gathered}
\left.-\left[\boldsymbol{L}^{(2)}\left(\beta_{i}^{(1)} \boldsymbol{W}_{i}^{(1)}\right)\right]\right)-v \sum_{i=1}^{N_{0}}\left[\boldsymbol{L}^{(2)}\left(\beta_{i}^{(1)} \boldsymbol{W}_{i}^{(1)}-\beta_{i}^{(2)} \boldsymbol{W}_{i}^{(2)}\right)\right] \\
-\left[\boldsymbol{N}^{(1)}\left(\boldsymbol{w}^{(1)}+\sum_{i=1}^{N_{0}} \beta_{i}^{(1)}\right)\right]+\left[\boldsymbol{N}^{(2)}\left(\boldsymbol{w}^{(2)}+\sum_{i=1}^{N_{0}} \beta_{i}^{(2)}\right)\right] \\
-\sum_{i=1}^{N_{0}}\left(\left[\boldsymbol{G}^{(1)}\left(\beta_{i}^{(1)} \pi_{i}^{(1)}\right)\right]-\left[\boldsymbol{G}^{(2)}\left(\beta_{i}^{(1)} \pi_{i}^{(1)}\right)\right]\right)-\sum_{i=1}^{N_{0}}\left[\boldsymbol{G}^{(2)}\left(\beta_{i}^{(1)} \pi_{i}^{(1)}-\beta_{i}^{(2)} \pi_{i}^{(2)}\right)\right] \\
-\left(\left[\boldsymbol{M}^{(1)} \boldsymbol{w}^{(1)}\right]-\left[\boldsymbol{M}^{(2)} \boldsymbol{w}^{(1)}\right]\right)-\left[\boldsymbol{M}^{(2)}\left(\boldsymbol{w}^{(1)}-\boldsymbol{w}^{(2)}\right)\right] \\
-v\left(\left[\boldsymbol{L}^{(1)} \boldsymbol{w}^{(1)}\right]-\left[\boldsymbol{L}^{(2)} \boldsymbol{w}^{(1)}\right]\right)-v\left(\left[\boldsymbol{L}^{(2)}\left(\boldsymbol{w}^{(1)}-\boldsymbol{w}^{(2)}\right)\right]-\boldsymbol{\Delta}\left(\boldsymbol{w}^{(1)}-\boldsymbol{w}^{(2)}\right)\right) \\
-\left(\left[\boldsymbol{G}^{(1)} \pi^{(1)}\right]-\left[\boldsymbol{G}^{(2)} \pi^{(1)}\right]\right)-\left(\left[\boldsymbol{G}^{(2)}\left(\pi^{(1)}-\pi^{(2)}\right)\right]-\nabla\left(\pi^{(1)}-\pi^{(2)}\right)\right),
\end{gathered}
$$

and

$$
\begin{aligned}
& f_{i}^{(1)}-f_{i}^{(2)}=\left(\beta_{i}^{(1)^{\prime}}-\beta_{i}^{(2)^{\prime}}\right) \int_{\Omega_{S}}\left(\widehat{\boldsymbol{\xi}}_{j}^{(1)}-\boldsymbol{\xi}_{j}\right) \cdot \boldsymbol{\xi}_{i} d \boldsymbol{y}+\beta_{j}^{(2)^{\prime}} \int_{\Omega_{S}}\left(\widehat{\boldsymbol{\xi}}_{j}^{(1)}-\widehat{\boldsymbol{\xi}}_{j}^{(2)}\right) \cdot \boldsymbol{\xi}_{i} d \boldsymbol{y} \\
& +\left(\beta_{i}^{(1)}-\beta_{i}^{(2)}\right) \int_{\Omega_{S}} \frac{\partial}{\partial t}\left(\widehat{\boldsymbol{\xi}}_{j}^{(1)}\right) \cdot \widehat{\boldsymbol{\xi}}_{i}^{(1)} d \boldsymbol{y}+\beta_{j}^{(2)} \int_{\Omega_{S}} \frac{\partial}{\partial t}\left(\widehat{\boldsymbol{\xi}}_{j}^{(1)}-\widehat{\boldsymbol{\xi}}_{j}^{(2)}\right) \cdot \widehat{\boldsymbol{\xi}}_{i}^{(1)} d \boldsymbol{y} \\
& +\beta_{j}^{(2)} \int_{\Omega_{S}} \frac{\partial}{\partial t}\left(\widehat{\boldsymbol{\xi}}_{j}^{(2)}\right) \cdot\left(\widehat{\boldsymbol{\xi}}_{i}^{(1)}-\widehat{\boldsymbol{\xi}}_{i}^{(2)}\right) d \boldsymbol{y}+2 \mu \int_{\Omega_{S}} \boldsymbol{\varepsilon}\left(\boldsymbol{\xi}^{(1)}-\boldsymbol{\xi}^{(2)}\right): \boldsymbol{\varepsilon}\left(\widehat{\boldsymbol{\xi}}_{i}^{(1)}\right) d \boldsymbol{y} \\
& +2 \mu \int_{\Omega_{S}} \boldsymbol{\varepsilon}\left(\xi^{(2)}\right): \boldsymbol{\varepsilon}\left(\widehat{\boldsymbol{\xi}}_{i}^{(1)}-\widehat{\boldsymbol{\xi}}_{i}^{(2)}\right) d \boldsymbol{y}+\lambda \int_{\Omega_{S}} \operatorname{div}\left(\xi^{(1)}-\boldsymbol{\xi}^{(2)}\right) \operatorname{div}\left(\widehat{\boldsymbol{\xi}}_{i}^{(1)}\right) d \boldsymbol{y} \\
& +\lambda \int_{\Omega_{S}} \operatorname{div}\left(\xi^{(2)}\right) \operatorname{div}\left(\widehat{\xi}_{i}^{(1)}-\widehat{\xi}_{i}^{(2)}\right) d \boldsymbol{y}+\int_{\partial \Omega_{S}}\left[\sigma\left(w^{(1)}, \pi^{(1)}\right)-\sigma\left(w^{(2)}, \pi^{(2)}\right)\right] n_{y} \cdot\left(\widehat{\xi}_{i}^{(1)}-\xi_{i}\right) d \gamma_{y} \\
& +\int_{\partial \Omega_{S}} \sigma\left(w^{(2)}, \pi^{(2)}\right) n_{y} \cdot\left(\widehat{\xi}_{i}^{(1)}-\widehat{\xi}_{i}^{(2)}\right) d \gamma_{y}+\int_{\partial \Omega_{S}}\left(G_{0}^{(1)}-G_{0}^{(2)}\right)\left[w^{(1)}, \pi^{(1)}\right] \cdot \widehat{\xi}_{i}^{(1)} d \gamma_{y} \\
& +\int_{\partial \Omega_{S}} \boldsymbol{G}_{0}\left[\left(\boldsymbol{w}^{(1)}, \pi^{(1)}\right)-\left(\boldsymbol{w}^{(2)}, \pi^{(2)}\right)\right] \cdot \widehat{\boldsymbol{\xi}}_{i}^{(1)} d \boldsymbol{\gamma}_{\boldsymbol{y}}+\int_{\partial \Omega_{S}} \boldsymbol{G}_{0}^{(2)}\left[\boldsymbol{w}^{(2)}, \pi^{(2)}\right] \cdot\left(\widehat{\xi}_{i}^{(1)}-\widehat{\boldsymbol{\xi}}_{i}^{(2)}\right) d \boldsymbol{\gamma}_{\boldsymbol{y}} .
\end{aligned}
$$

From the above equalities and Lemmata 6.1-6.2, 6.4, 7.1-7.4, we deduce (68). This concludes the proof of Theorem 5.2.

Acknowledgements. The authors were partially supported by the Agence Nationale de la Recherche, Project CISIFS, grant ANR-09-BLAN-0213-02.

\section{REFERENCES}

1. Boulakia, M., Existence of weak solutions for the three-dimensional motion of an elastic structure in an incompressible fluid, J. Math. Fluid Mech. 9 (2007), 262-294. Zbl1171.74337 MR2329269

2. Cattabriga, L., Su un problema al contorno relativo al sistema di equazioni di Stokes, Rend. Sem. Mat. Univ. Padova 31 (1961), 308-340. Zb10116.18002 MR0138894

3. Ciarlet, P. G., Mathematical elasticity. Vol. I, vol. 20 of Studies in Mathematics and its Applications, North-Holland Publishing Co., Amsterdam, 1988. Three-dimensional elasticity. Zb10648.73014 MR0936420 
4. Conca, C., San Martín H., J., \& Tucsnak, M., Existence of solutions for the equations modelling the motion of a rigid body in a viscous fluid, Comm. Partial Differential Equations 25 (2000), 1019-1042. Zb10954.35135 MR1759801

5. Coutand, D. \& ShKoller, S., Motion of an elastic solid inside an incompressible viscous fluid, Arch. Ration. Mech. Anal. 176 (2005), 25-102. Zbl1064.74057 MR2185858

6. Cumsille, P. \& TAKahashi, T., Wellposedness for the system modelling the motion of a rigid body of arbitrary form in an incompressible viscous fluid, Czechoslovak Math. J. 58 (2008), 961-992. Zbl1174. 35092 MR2471160

7. Desjardins, B., Esteban, M. J, Grandmont, C., \& Le Tallec, P., Weak solutions for a fluidelastic structure interaction model, Rev. Mat. Complut. 14 (2001), 523-538. Zb11007. 35055 MR1871311

8. Du, Q., Gunzburger, M. D., Hou, L. S., \& LeE, J., Analysis of a linear fluid-structure interaction problem, Discrete Contin. Dyn. Syst. 9 (2003), 633-650. Zbl1039. 35076 MR1974530

9. Evans, L. C., Partial differential equations, vol. 19 of Graduate Studies in Mathematics, American Mathematical Society, Providence, RI, 1998. Zb10902.35002 MR1625845

10. Feireisl, E., On the motion of rigid bodies in a viscous incompressible fluid, J. Evol. Equ. 3 (2003), 419-441. Dedicated to Philippe Bénilan. Zbl1039.76071 MR2019028

11. GRAndmont, C., Existence of weak solutions for the unsteady interaction of a viscous fluid with an elastic plate, SIAM J. Math. Anal. 40 (2008), 716-737. Zbl1158.74016 MR2438783

12. Gunzburger, M. D., Lee, H.-C., \& Seregin, G. A., Global existence of weak solutions for viscous incompressible flows around a moving rigid body in three dimensions, J. Math. Fluid Mech. 2 (2000), 219-266. Zb10970.35096 MR1781915

13. Hillairet, M., Lack of collision between solid bodies in a 2D incompressible viscous flow, Comm. Partial Differential Equations 32 (2007), 1345-1371. Zbl1221. 35279 MR2354496

14. Raviart, P.-A. \& Thomas, J.-M., Introduction à l'analyse numérique des équations aux dérivées partielles, Collection Mathématiques Appliquées pour la Maîtrise. [Collection of Applied Mathematics for the Master's Degree], Masson, Paris, 1983. Zb10561.65069 MR0773854

15. San Martín, J. A., Starovoitov, V., \& Tucsnak, M., Global weak solutions for the twodimensional motion of several rigid bodies in an incompressible viscous fluid, Arch. Ration. Mech. Anal. 161 (2002), 113-147. Zbl1018.76012 MR1870954

16. TAKAHASHI, T., Analysis of strong solutions for the equations modeling the motion of a rigid-fluid system in a bounded domain, Adv. Differential Equations 8 (2003), 1499-1532. Zbl1101. 35356 MR2029294

17. TuCsnak, M. \& Weiss, G., Observation and control for operator semigroups, Birkhäuser Advanced Texts: Basler Lehrbücher. [Birkhäuser Advanced Texts: Basel Textbooks], Birkhäuser Verlag, Basel, 2009. Zbl1188.93002 MR2588549 